\title{
Systematic Review \\ Understanding the Spectrum of Anxiety Responses to Climate Change: A Systematic Review of the Qualitative Literature
}

\author{
Catriona Soutar ${ }^{1, *}$ and Anne P. F. Wand ${ }^{2,3} \mathbb{D}$ \\ 1 Sydney Local Health District, Sydney 2050, Australia \\ 2 Specialty of Psychiatry, Faculty of Medicine and Health, University of Sydney, Sydney 2050, Australia; \\ anne.wand@sydney.edu.au \\ 3 School of Psychiatry, Faculty of Medicine, University of New South Wales, Sydney 2033, Australia \\ * Correspondence: catriona.soutar@health.nsw.gov.au; Tel.: +61-295-159-000
}

Citation: Soutar, C.; Wand, A.P.F. Understanding the Spectrum of Anxiety Responses to Climate Change: A Systematic Review of the Qualitative Literature. Int. J. Environ. Res. Public Health 2022, 19, 990.

https://doi.org/10.3390/

ijerph19020990

Academic Editor: Alan E. Stewart

Received: 6 December 2021

Accepted: 13 January 2022

Published: 16 January 2022

Publisher's Note: MDPI stays neutral with regard to jurisdictional claims in published maps and institutional affiliations.

Copyright: (c) 2022 by the authors. Licensee MDPI, Basel, Switzerland. This article is an open access article distributed under the terms and conditions of the Creative Commons Attribution (CC BY) license (https:// creativecommons.org/licenses/by/ $4.0 /)$.

\begin{abstract}
Background: Knowledge about climate change may produce anxiety, but the concept of climate change anxiety is poorly understood. The primary aim of this study was to systematically review the qualitative literature regarding the scope of anxiety responses to climate change. The secondary aim was to investigate the sociodemographic and geographical factors which influence experiences of climate change anxiety. Methods: A systematic review of empirical qualitative studies was undertaken, examining the scope of climate change anxiety by searching five databases. Studies were critically appraised for quality. Content analysis was used to identify themes. Results: Fifteen studies met the inclusion criteria. The content analysis was organised into two overarching themes. The scope of anxiety included worry about threats to livelihood, worry for future generations, worry about apocalyptic futures, anxiety at the lack of response to climate change, and competing worries. Themes pertaining to responses to climate change anxiety included symptoms of anxiety, feeling helpless and disempowered, and ways of managing climate change anxiety. Relatively few studies were identified, with limited geographical diversity amongst the populations studied. Conclusions: The review furthers understanding of the concept of climate change anxiety and responses to it, highlighting the need for high-quality psychiatric research exploring its clinical significance and potential interventions.
\end{abstract}

Keywords: anxiety; climate change; global warming; qualitative measures; eco-anxiety

\section{Introduction}

Climate change has been identified as one of the most pressing issues of our time, with major impacts on both physical and mental health [1]. Beyond the direct challenges that climate change poses to the determinants of mental health, such as threats to accessing basic needs (such as water, fresh air, food, and stable housing) and the trauma associated with extreme weather events, the broader psychological and emotional effects of climate change are increasingly being recognised [2-4]. This emerging field of study includes the interrelated phenomena of solastalgia, eco-anxiety, and ecological grief. Solastalgia, or the distress produced by the degradation of one's home environment, was a concept first introduced by Albrecht [5], and it has been the subject of a growing body of literature since [6,7]. Ecological grief is a related concept, relating to grief experienced at ecological losses, i.e., to the loss of species, ecosystems, and meaningful landscapes [8,9]. Eco-anxiety has been defined by the American Psychological Association as "a chronic fear of environmental doom" [10].

Climate change anxiety has been proposed as the most recognised form of ecoanxiety [11]. It has been defined as "anxiety associated with perceptions about climate change"(p. 2) [12], which may involve cognitive, emotional, or functional impairment and somatic arousal (bodily symptoms) [13]. A related concept, climate change worry, 
involves negative or apprehensive thoughts about climate change that may be repetitive, difficult to control, or persistent [14]. Thus, worry may be a component of climate change anxiety. In recent years, climate change anxiety has become topical within mainstream media $[15,16]$. Climate change was the leading worry for Australians in a recent national media survey [17], and up to $80 \%$ of young people report being somewhat or very anxious about climate change [18]. Climate change anxiety has also been well recognised within the academic literature [11,12] and formal studies have found a similar prevalence of anxiety as the media surveys [19]. Despite increasing recognition, climate change anxiety remains an emerging concept that is in the early stages of being understood [12]. To date, we are not aware of any studies that have systematically examined the breadth of experiences comprising climate change anxiety, which is essential to furthering our understanding of this concept.

The aim of this systematic review is to explore the empirical qualitative literature examining the scope of climate change anxiety and the spectrum of responses to it. A secondary aim is to explore the factors influencing climate change anxiety. Qualitative methodologies are most appropriate to develop an in-depth understanding of a new phenomenon, as they explore subjective experiences and narratives in an open way [20]. Thus, this review focuses on studies using qualitative approaches in order to richly describe the nature and scope of anxiety in response to climate change.

\section{Materials and Methods}

\subsection{Search Strategy}

The review was conducted according to PRISMA reporting guidelines. A search of the published literature was performed using the databases Medline, PsychINFO, ProQuest, Web of Science, and Scopus. Search terms used were 'climate change' OR 'global warming' combined with 'anxiety' OR 'worry' OR 'fear' OR 'eco-anxiety'. MESH Headings were utilised where possible. The search was limited to English-language papers utilising qualitative methodology to examine the psychological/emotional experiences of, and responses to, climate change that fell within the anxiety spectrum. Only empirical research published in peer-reviewed journals from January 2000 to August 22nd, 2020, was included. Duplicate citations were removed after the initial search.

The results of the database searches were initially screened by title and abstract by the first author. All articles potentially meeting the inclusion criteria (described below) were reviewed in full text. Where it was unclear whether an article met the inclusion criteria, it was discussed with the co-author until a consensus was reached.

To maximise the comprehensiveness of the search, citation chaining was performed on all papers that met the inclusion criteria to identify further relevant papers. In addition, three experts in the field of climate change and mental health were contacted for further recommendations.

\subsection{Inclusion and Exclusion Criteria}

Any studies that documented anxiety-related emotional or psychological experiences (e.g., anxiety, fear, worry) in relation to climate change using qualitative methodology (including mixed methods) were eligible for inclusion. Papers were included regardless of whether anxiety about climate change was the primary or secondary focus of the study, or an emergent finding. Studies of climate change worry, a component of climate change anxiety, were eligible for inclusion if they examined the nature and content of anxious cognitions about climate change.

Papers that examined anxiety responses to individual environmental phenomena (e.g., natural disasters, sea level rises, or environmental change within a specific landscape) were excluded. Although related, they are considered to be different phenomena to anxiety in response to the knowledge of climate change, which was the focus of this study. Papers studying perceptions of climate change that did not identify anxiety responses were also 
excluded, as were papers reporting only quantitative data. Opinion pieces, reviews, and grey literature were excluded from the review.

\subsection{Quality Rating}

Each paper that met the inclusion criteria was assigned a quality rating as per the guideline checklist developed by Attree and Milton [21]. This guideline describes a systematic rating scale for qualitative research papers. Each study is rated on criteria that include research background, aims and objectives, context, appropriateness of design, sampling, data collection and analysis, reflexivity, value of the research, and ethics. A total score is assigned based on the grade for the majority of sections. Studies receiving ' $A$ ' scores have no or few flaws, ' $\mathrm{B}$ ' have some flaws, ' $\mathrm{C}$ ' have considerable flaws but are still of some value, and ' $\mathrm{D}$ ' scores have significant flaws that threaten the validity of the study as a whole.

Each paper was analysed and scored independently by both authors. Where assigned scores differed, there was discussion of ratings until a consensus was reached. As per Attree and Milton's recommendations, papers with a score of $\mathrm{D}$ were excluded from the analysis [21].

\subsection{Data Extraction and Thematic Analysis}

Data were extracted independently by both authors. The standardised form for data extraction included study details (author, year, country of study), aim, design and methodology, participants and setting, anxiety themes identified, other themes identified, factors influencing the emotional or psychological experience of climate change, and quality rating, with comments on methodological factors.

A thematic analysis was conducted by the lead author. The methodology followed the six phases described by Braun and Clarke [22], using a process of inductive analysis. Using this technique, data are coded and themes are derived directly from the data presented, without pre-conceived themes and with a broad research question [22]. The phases included familiarisation with the data by reading and re-reading the texts, generating initial codes, searching for themes, reviewing themes, defining and naming themes, and producing the report. Emergent themes were discussed with the co-author until a consensus was reached. Themes were revised iteratively in light of new data until the final themes and sub-themes were established.

\section{Results}

The PRISMA diagram outlines the search process and selection of papers (see Figure 1).

\subsection{Study Characteristics}

A total of 15 studies met the inclusion criteria [23-37]. The study information and detailed findings are presented in Table 1. Four studies were conducted in North America $[24,26,29,30]$, three in Australia [25,33,35], two in Norway [31,34], and one each from South Korea [23], Sweden [37], Ghana [32], and Tuvalu [27]. One study was conducted across four nations (Fiji, Cyprus, New Zealand, and the United Kingdom) [36] and one study did not identify its origin [28]. The majority of studies (10/15) were published in the previous five years. All but one [37] focused on adults, though the two Norwegian studies included a small number of teenagers within groups of predominantly adult participants $[31,34]$. 


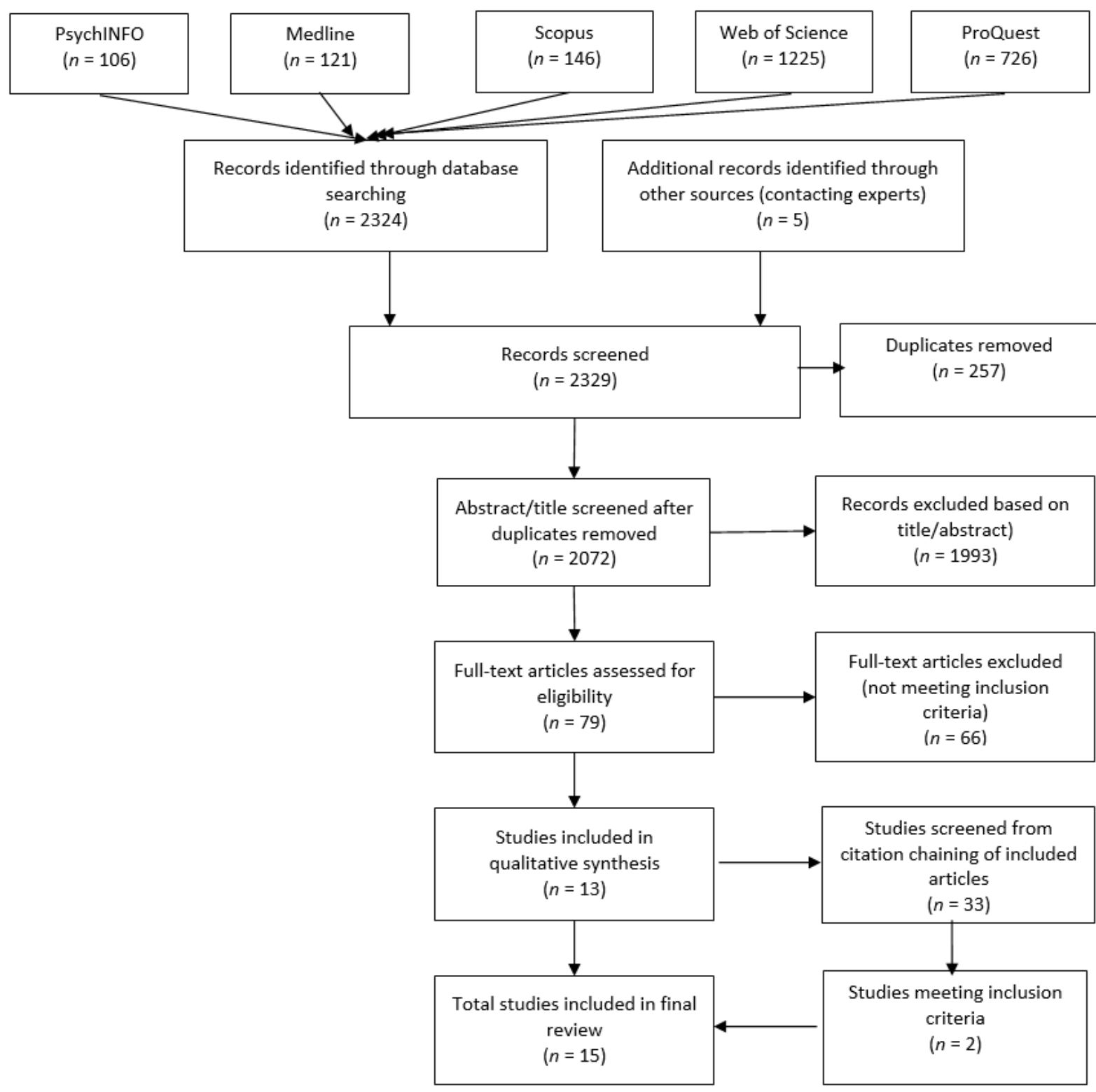

Figure 1. PRISMA flow diagram.

Six of the papers were purely qualitative and the other nine used mixed methods (see Table 1). The stated goals of the studies were variable; nine sought to study mental wellbeing and emotions in the context of climate change [24,27-31,35-37], and six explored broader perceptions of climate change [23,25,26,32-34]. Two studies included quantitative measures of distress and anxiety [27,29]. Five studies predominantly investigated for, or identified, climate change anxiety [23,27-29,35], whereas ten studies focused on responses more consistent with climate change worry [24-26,30-34,36,37].

Five studies scored the highest quality rating of $A$, five were rated $B$ and four were rated $C$ (see Table 1). A single study [31] was rated D and was thus excluded from the analysis. 
Table 1. Description of included studies, thematic analysis, and quality ratings.

\begin{tabular}{|c|c|c|c|c|c|c|c|}
\hline $\begin{array}{c}\text { Author, Year, } \\
\text { Country of Study }\end{array}$ & Design and Methodology & Aim & Participants and Setting & $\begin{array}{l}\text { Anxiety Themes } \\
\text { Identified (Including } \\
\text { Themes about Worry } \\
\text { Where Relevant) }\end{array}$ & Other Themes Identified & $\begin{array}{l}\text { Factors Influencing } \\
\text { Experience of Climate } \\
\text { Change }\end{array}$ & $\begin{array}{l}\text { Quality Rating and } \\
\text { Comments }\end{array}$ \\
\hline $\begin{array}{l}\text { Anghelcev et al. } \\
2015 \\
\text { South Korea }\end{array}$ & $\begin{array}{l}\text { Semi-structured interviews } \\
\text { and photo elicitation } \\
\text { Zaltman metaphor } \\
\text { elicitation technique } \\
\text { (ZMET) and narrative } \\
\text { thematic analysis }\end{array}$ & $\begin{array}{l}\text { Primary: to illustrate the } \\
\text { applicability of using } \\
\text { ZMET in social marketing } \\
\text { communication } \\
\text { Secondary: to explore how } \\
\text { climate change is perceived } \\
\text { by young members of the } \\
\text { Korean public }\end{array}$ & $\begin{array}{l}n=12(\mathrm{M} 6, \mathrm{~F} 6) \\
\text { Age range 20-28 } \\
\text { South Korean college } \\
\text { students } \\
\text { Half undergraduate and } \\
\text { half graduate }\end{array}$ & $\begin{array}{l}\text { Affective distress (fear, } \\
\text { nostalgia, sadness) was } \\
\text { one of } 3 \text { "deep metaphors" } \\
\text { found } \\
\text { Fear: apocalyptic futures } \\
\text { (doomsday scenarios } \\
\text { promoted by media) } \\
\text { Fear accompanied by } \\
\text { anxiety symptoms (feeling } \\
\text { of suffocation, inevitable } \\
\text { destruction) }\end{array}$ & $\begin{array}{l}\text { Sadness (loss of world as we } \\
\text { know it, futility of individual } \\
\text { action and inability to reverse } \\
\text { climate change) } \\
\text { Nostalgia (memories of idealised } \\
\text { past) } \\
\text { Pandora's box } \\
\text {-tragic endings (loss of } \\
\text { biodiversity and human habitat) } \\
\text { - human greed (corporate greed } \\
\text { for profits, selfish pursuit of } \\
\text { comfort and gratification) } \\
\text {-hope (education as agent of } \\
\text { positive change, stricter } \\
\text { governmental control) } \\
\text { Two-faced Janus } \\
\text { - discursive ambivalence (dual } \\
\text { standards of accountability, } \\
\text { ambivalent media discourse) } \\
\text { - functional duality (technology as } \\
\text { cause and solution, home as space } \\
\text { of consumption and mitigation) }\end{array}$ & $\begin{array}{l}\text { Participants perceived } \\
\text { climate change as } \\
\text { something geographically } \\
\text { or temporally distant (not } \\
\text { a direct threat or } \\
\text { happening to them) }\end{array}$ & $\begin{array}{l}\text { B } \\
\text {-No information on ethical } \\
\text { considerations } \\
\text {-Bias in sampling (personal } \\
\text { solicitation) } \\
\text {-Quotes not matched to } \\
\text { demographic data } \\
\text {-No respondent validation } \\
\text {-No reflexivity or } \\
\text { consideration of cultural } \\
\text { factors }\end{array}$ \\
\hline $\begin{array}{l}\text { Du Bray et al. } \\
2017 \\
\text { Fiji, Cyprus, New } \\
\text { Zealand, United } \\
\text { Kingdom }\end{array}$ & $\begin{array}{l}\text { Open-ended individual } \\
\text { ethnographic interviews } \\
\text { Interviews autocoded for } \\
\text { positive and negative } \\
\text { emotion words (counting } \\
\text { of intext references) } \\
\text { The method of qualitative } \\
\text { data analysis of interview } \\
\text { texts was not described }\end{array}$ & $\begin{array}{l}\text { "To understand how } \\
\text { emotional responses to } \\
\text { climate change are } \\
\text { inequitably distributed } \\
\text { across people living in } \\
\text { island nations with } \\
\text { varying climate change } \\
\text { vulnerability" (p. 1) }\end{array}$ & $\begin{array}{l}n=272 \\
\text { Residents in } 4 \text { island } \\
\text { nations: Fiji }(n=68), \\
\text { Cyprus }(n=40) \text {, New } \\
\text { Zealand }(n=86) \text {, United } \\
\text { Kingdom }(n=78) \\
\text { Gender and ages not } \\
\text { provided }\end{array}$ & $\begin{array}{l}\text { Fiji: worry about land, } \\
\text { people, community, plants } \\
\text { not growing, loss of } \\
\text { self-sufficiency and } \\
\text { cultural traditions } \\
\text { Cyprus: worry about the } \\
\text { future, rise of sea level, } \\
\text { water scarcity, how to } \\
\text { adapt to change } \\
\text { New Zealand: worry about } \\
\text { friends and family, speed } \\
\text { of change, rise of global } \\
\text { disasters } \\
\text { UK: worry about } \\
\text { grandchildren, population } \\
\text { changes (incoming } \\
\text { refugees) }\end{array}$ & $\begin{array}{l}\text { Fiji: happiness and sorrow, pride } \\
\text { in cultural heritage, sad at loss of } \\
\text { livelihood traditions, new } \\
\text { opportunities with relocation of } \\
\text { younger generation } \\
\text { Cyprus: concern/frustration with } \\
\text { water shortage, } \\
\text { anger/helplessness. Sadness } \\
\text { about rainfall changes, unable to } \\
\text { trust local agriculture } \\
\text { NZ: sad at ecological loss. Hope } \\
\text { for future generations, optimism } \\
\text { UK: neutral, felt would not be } \\
\text { impacted, cannot control climate } \\
\text { change, therefore must not } \\
\text { become upset about it }\end{array}$ & $\begin{array}{l}\text { Island nations are } \\
\text { vulnerable to climate } \\
\text { change to varying degrees } \\
\text { (UK and NZ less } \\
\text { vulnerable, more adaptive } \\
\text { capacity, Cyrus and Fiji } \\
\text { more vulnerable with less } \\
\text { adaptive capacity) }\end{array}$ & $\begin{array}{l}\text { C } \\
\text {-Poor description of context } \\
\text {-Insufficient demographic } \\
\text { information on } \\
\text { participants } \\
\text {-Sampling strategy not } \\
\text { documented } \\
\text {-No justification of sample } \\
\text { size/data saturation } \\
\text {-Data recording method } \\
\text { unclear } \\
\text {-Qualitative data analysis } \\
\text { not recorded } \\
\text {-No validation of findings } \\
\text {-No consideration of } \\
\text { limitations } \\
\text {-No reflexivity or ethics } \\
\text { considered }\end{array}$ \\
\hline
\end{tabular}


Table 1. Cont.

\begin{tabular}{|c|c|c|c|c|c|c|c|}
\hline $\begin{array}{l}\text { Author, Year, } \\
\text { Country of Study }\end{array}$ & Design and Methodology & Aim & Participants and Setting & $\begin{array}{l}\text { Anxiety Themes } \\
\text { Identified (Including } \\
\text { Themes about Worry } \\
\text { Where Relevant) }\end{array}$ & Other Themes Identified & $\begin{array}{c}\text { Factors Influencing } \\
\text { Experience of Climate } \\
\text { Change }\end{array}$ & $\begin{array}{l}\text { Quality Rating and } \\
\text { Comments }\end{array}$ \\
\hline $\begin{array}{l}\text { Du Bray et al. } \\
2017 \\
\text { USA }\end{array}$ & $\begin{array}{l}\text { Participant observation } \\
\text { and mixed-method survey } \\
\text { (16 open ended interview } \\
\text { questions and } 21 \text { survey } \\
\text { questions) } \\
\text { Counting and coding of } \\
\text { emotion words (KWIC } \\
\text { (keywords-in-context) } \\
\text { approach) } \\
\text { Themes emerged } \\
\text { deductively from the } \\
\text { pattern of questioning } \\
\text { The method of analysis is } \\
\text { unclear }\end{array}$ & $\begin{array}{l}\text { Primary: to determine } \\
\text { "how emotional responses } \\
\text { to climate change vary } \\
\text { across sites with different } \\
\text { experiences and projected } \\
\text { outcomes as a result of } \\
\text { climate change" (p. 286) } \\
\text { Secondary: to determine } \\
\text { "whether men or women } \\
\text { were more likely to express } \\
\text { emotions across these three } \\
\text { sites" (p. 286) }\end{array}$ & $\begin{array}{l}n=103 \text { (M 50, F 53) } \\
\text { Ages not provided } \\
\text { Residents of } 3 \text { US cities: } \\
\text { Mobile, Alabama (31), } \\
\text { Kodiak, Alaska (36), and } \\
\text { Phoenix, Arizona (36) }\end{array}$ & $\begin{array}{l}\text { Worry and sadness for } \\
\text { future generations } \\
\text { Worry about financial } \\
\text { issues/loss of livelihood } \\
\text { (access to resources) or } \\
\text { being unable to give } \\
\text { children same livelihood } \\
\text { opportunities } \\
\text { Concern about changes to } \\
\text { landscape } \\
\text { Lack of worry in those } \\
\text { who believe that climate } \\
\text { change does not affect } \\
\text { current generation } \\
\text { Site specific: } \\
\text { Alabama-worry for } \\
\text { others but more worried } \\
\text { by other natural events } \\
\text { (hurricanes, storm water } \\
\text { runoff) which will be made } \\
\text { worse with climate change. } \\
\text { Uncertainty and } \\
\text { adaptation } \\
\text { Alaska-anxiety was not a } \\
\text { prominent theme } \\
\text { Arizona-less emotional } \\
\text { about climate change } \\
\text { overall. Concern for } \\
\text { younger generation (not } \\
\text { have same experience) }\end{array}$ & $\begin{array}{l}\text { Alabama: } \\
\text { Large number of respondents did } \\
\text { not believe in anthropogenic } \\
\text { climate change } \\
\text { Participants felt more prepared, } \\
\text { able to adapt than other sites } \\
\text { Alaska: } \\
\text { Many respondents believed in } \\
\text { anthropogenic climate change, } \\
\text { (31\% did not) } \\
\text { Participants more likely to express } \\
\text { hope for future, optimism or } \\
\text { feeling safe, though there are } \\
\text { others who anticipate negative } \\
\text { consequences } \\
\text { Some anticipated positive changes } \\
\text { Arizona: } \\
\text { Participants were least likely to } \\
\text { indicate emotional reaction to } \\
\text { climate change } \\
\text { Resignation, unconcerned, feeling } \\
\text { climate change is inevitable }\end{array}$ & $\begin{array}{l}\text { USA less immediately } \\
\text { impacted by climate } \\
\text { change } \\
\text { Alabama: } \\
\text { Susceptible to hurricanes, } \\
\text { coastal vulnerability, } \\
\text { fishing/agriculture } \\
\text { reliance } \\
\text { Alaska: } \\
\text { Fishing/wilderness } \\
\text { reliance, Indigenous way } \\
\text { of life is vulnerable to } \\
\text { climate change } \\
\text { Arizona: } \\
\text { Urbanised environment, } \\
\text { buffered from local ecology } \\
\text { Gender: women more } \\
\text { likely than men to evoke } \\
\text { "worry". Men worried } \\
\text { about financial survival } \\
\text { and livelihood. Women } \\
\text { worried about future } \\
\text { generations }\end{array}$ & $\begin{array}{l}\text { B } \\
\text {-Sampling strategy poorly } \\
\text { described } \\
\text {-Data collection and } \\
\text { recording unclear } \\
\text {-Method of analysis } \\
\text { (counting keywords) is not } \\
\text { the most appropriate to } \\
\text { answer the study question } \\
\text { in depth } \\
\text {-Few quotes, not matched } \\
\text { to demographic data } \\
\text {-No validation of findings } \\
\text {-No discussion of } \\
\text { reflexivity or limitations } \\
\text {-Good demographic } \\
\text { description } \\
\text {-Content validity through } \\
\text { pre-testing } \\
\text {-Some triangulation in data } \\
\text { collection (field notes, } \\
\text { participant observation, } \\
\text { interviews) } \\
\text {-Trustworthiness through } \\
\text { deliberate selection of } \\
\text { similar and different views }\end{array}$ \\
\hline $\begin{array}{l}\text { Fleming et al } \\
2015 \\
\text { Australia }\end{array}$ & $\begin{array}{l}\text { Mixed-method telephone } \\
\text { survey (open-ended and } \\
\text { Likert-scaled responses) } \\
\text { and literature review } \\
\text { Qualitative analysis using } \\
\text { constructivist } \\
\text { interpretations of } \\
\text { grounded theory; used } \\
\text { NVivo9 software } \\
\text { Quantitative analysis: } \\
\text { scaled responses were } \\
\text { incorporated into codes, } \\
\text { categories, and themes }\end{array}$ & $\begin{array}{l}\text { To examine "how grape } \\
\text { growers in this region } \\
\text { perceive and prioritise } \\
\text { climate change adaptation } \\
\text { as an issue for their } \\
\text { industry" }\end{array}$ & $\begin{array}{l}n=50 \\
\text { Gender and ages not } \\
\text { provided } \\
\text { Grape growers in South } \\
\text { Australia } \\
50 / 68=74 \% \text { response rate }\end{array}$ & $\begin{array}{l}\text { Grape growers who were } \\
\text { sceptical about climate } \\
\text { change did not feel it } \\
\text { would bring risks or } \\
\text { opportunities } \\
\text { Those who were convinced } \\
\text { of it perceived greater risks }\end{array}$ & $\begin{array}{l}58 \% \text { were sceptical of } \\
\text { anthropogenic climate change } \\
\text { Focus of worry not related to } \\
\text { climate change included themes } \\
\text { of: } \\
\text { Significant concerns } \\
\text {-immediate stress (cash flow, lack } \\
\text { of succession, dwindling } \\
\text { communities with limited labour } \\
\text { access, lack of transparency) } \\
\text {-loss of enjoyment in lifestyle } \\
\text { Perceptions of climate change: } \\
\text { scepticism } \\
\text { Coping with stress } \\
\text {-committed farmer } \\
\text {-exiting the industry } \\
\text {-positive outliers }\end{array}$ & $\begin{array}{l}\text { High degree of scepticism } \\
\text { about climate change } \\
\text { influenced perception of } \\
\text { risks and tendency } \\
\text { towards action }\end{array}$ & $\begin{array}{l}\text { A } \\
\text {-Good response rate } \\
\text {-Quality content analysis } \\
\text {-Ethics approval } \\
\text {-Identification of outliers } \\
\text { validates results } \\
\text {-Data collection not well } \\
\text { recorded } \\
\text {-No data saturation } \\
\text {-Limited demographic data } \\
\text { provided } \\
\text {-No reflexivity discussed }\end{array}$ \\
\hline
\end{tabular}


Table 1. Cont.

\begin{tabular}{|c|c|c|c|c|c|c|c|}
\hline $\begin{array}{c}\text { Author, Year, } \\
\text { Country of Study }\end{array}$ & Design and Methodology & Aim & Participants and Setting & $\begin{array}{c}\text { Anxiety Themes } \\
\text { Identified (Including } \\
\text { Themes about Worry } \\
\text { Where Relevant) }\end{array}$ & Other Themes Identified & $\begin{array}{c}\text { Factors Influencing } \\
\text { Experience of Climate } \\
\text { Change }\end{array}$ & $\begin{array}{l}\text { Quality Rating and } \\
\text { Comments }\end{array}$ \\
\hline $\begin{array}{l}\text { Galway } \\
2019 \\
\text { Canada }\end{array}$ & $\begin{array}{l}\text { Semi-structured walking } \\
\text { interviews } \\
\text { Thematic analysis }\end{array}$ & $\begin{array}{l}\text { "(1) To examine how } \\
\text { community members of } \\
\text { Thunder Bay understand, } \\
\text { and think about the issue } \\
\text { of climate change; (2) To } \\
\text { examine how community } \\
\text { members of Thunder Bay } \\
\text { perceive climate change } \\
\text { impacts and action; and (3) } \\
\text { To consider the role of } \\
\text { place in relation to climate } \\
\text { change perceptions in the } \\
\text { context of Thunder Bay" } \\
\text { (p. 69) }\end{array}$ & $\begin{array}{l}n=18 \text { (M 8, F 10) } \\
\text { Ages 20s-70s } \\
\text { Residents in a remote city } \\
\text { in Northern Ontario, } \\
\text { Canada }\end{array}$ & $\begin{array}{l}\text { Fear and concern/worry } \\
\text { were most commonly } \\
\text { reported emotions } \\
\text { Worry for children and } \\
\text { future generations } \\
\text { Worry about future access } \\
\text { to water, food, forests }\end{array}$ & $\begin{array}{l}\text { Climate change as complex and } \\
\text { interconnected } \\
\text { Causes of climate change (fossil } \\
\text { fuel industry, industrialisation, } \\
\text { capitalism, greed, etc.) } \\
\text { Climate (in)justice and ethics } \\
\text { (intergenerational, marginalised } \\
\text { communities) } \\
\text { Taking notice of changes in the } \\
\text { weather, seasons and extreme } \\
\text { events } \\
\text { Anticipated future impacts on } \\
\text { water, food and forests } \\
\text {-Perceptions shaped by } \\
\text { experiences on land and water } \\
\text {-Transformation at a range of } \\
\text { levels, by a range of actors is } \\
\text { needed to address climate change } \\
\text { Other emotions included hopeful, } \\
\text { frustrated, sense of urgency, } \\
\text { depressed, angry/upset, guilt, sad }\end{array}$ & $\begin{array}{l}\text { The importance of place, } \\
\text { connection to local ecology } \\
\text { Local and regional settings } \\
\text { and relationships to this } \\
\text { land/ water/outdoors } \\
\text { Extreme flood } 2012 \\
\text { repeatedly referenced by } \\
\text { participants }\end{array}$ & $\begin{array}{l}\text { B } \\
\text {-No data saturation to } \\
\text { justify sample size } \\
\text {-No triangulation } \\
\text {-Quotes not matched to } \\
\text { demographic data } \\
\text {-Only one person analysing } \\
\text { data } \\
\text {-No validation of findings } \\
\text {-Little discussion of } \\
\text { limitations or alternative } \\
\text { explanations } \\
\text {-No reflexivity } \\
\text {-Themes not explicitly } \\
\text { presented } \\
\text {-Questions pilot tested for } \\
\text { clarity }\end{array}$ \\
\hline $\begin{array}{l}\text { Gibson et al } \\
2020 \\
\text { Tuvalu }\end{array}$ & $\begin{array}{l}\text { Mixed methods: structured } \\
\text { interview with open and } \\
\text { closed questions } \\
\text { Questionnaire: culturally } \\
\text { adapted Hopkins } \\
\text { Symptom Checklist-25 } \\
\text { Quantitative analysis: } \\
\text { descriptive, correlational, } \\
\text { and between-group } \\
\text { analysis } \\
\text { Qualitative analysis: } \\
\text { method poorly described }\end{array}$ & $\begin{array}{l}\text { To determine if residents in } \\
\text { Tuvalu report distress on } \\
\text { account of both local and } \\
\text { abstract climate change } \\
\text { To examine the extent to } \\
\text { which reported distress } \\
\text { impacts on daily } \\
\text { functioning }\end{array}$ & $\begin{array}{l}n=100 \text { (M 50, F 50) } \\
\text { Ages 18-24 (23), 25-39 (26), } \\
40-54 \text { (25), 55+(26) } \\
\text { Community members } \\
\text { living on Funafuti atoll, } \\
\text { Tuvalu }\end{array}$ & $\begin{array}{l}\text { Quantitative } \\
76 \% \% \text { reported worry about } \\
\text { abstract climate stressors } \\
\text { Of those with distress, } 79 \% \\
\text { reported impairment in } \\
\text { daily life and } 28 \% \text { reported } \\
\text { extreme impairment } \\
\text { Qualitative } \\
\text { Worry about safety, lack of } \\
\text { disaster preparedness, } \\
\text { having nowhere to go } \\
\text { Impact of worry on daily } \\
\text { function: poor sleep due to } \\
\text { climate change thoughts, } \\
\text { not going out, disturbs } \\
\text { leisure time }\end{array}$ & $\begin{array}{l}\text { Sadness prominent: } 79 \% \text { for local } \\
\text { aspects and } 77 \% \text { for abstract } \\
\text { climate change } \\
\text { Approx. } 84 \% \text { reported } \\
\text { worry/anxiety in response to } \\
\text { local climate change stressors, and } \\
79 \% \text { reported sadness about } \\
\text { environmental impacts, loss of } \\
\text { homes and decreasing capacity to } \\
\text { grow crops } \\
\text { Distress and anxiety related to } \\
\text { local climate stressors were more } \\
\text { common than to abstract climate } \\
\text { change }\end{array}$ & $\begin{array}{l}\text { Extremely vulnerable } \\
\text { country which may } \\
\text { become uninhabitable due } \\
\text { to sea level rise } \\
\text { Awareness of this reality is } \\
\text { found throughout Tuvalu } \\
\text { Poverty: those with more } \\
\text { financial hardship reported } \\
\text { greater distress } \\
\text { Distress attributed to } \\
\text { climate change (local and } \\
\text { abstract) showed } \\
\text { small-moderate } \\
\text { correlations with } \\
\text { psychological distress } \\
\text { more broadly }\end{array}$ & $\begin{array}{l}\text { B } \\
\text {-No respondent validation } \\
\text {-Little reflexivity } \\
\text {-No clear methodology } \\
\text { presented for qualitative } \\
\text { analysis } \\
\text {-Themes not explicitly } \\
\text { presented } \\
\text {-Questions were piloted } \\
\text { locally, assessed for } \\
\text { internal consistency }\end{array}$ \\
\hline
\end{tabular}


Table 1. Cont.

\begin{tabular}{|c|c|c|c|c|c|c|c|}
\hline $\begin{array}{l}\text { Author, Year, } \\
\text { Country of Study }\end{array}$ & Design and Methodology & Aim & Participants and Setting & $\begin{array}{l}\text { Anxiety Themes } \\
\text { Identified (Including } \\
\text { Themes about Worry } \\
\text { Where Relevant) }\end{array}$ & Other Themes Identified & $\begin{array}{l}\text { Factors Influencing } \\
\text { Experience of Climate } \\
\text { Change }\end{array}$ & $\begin{array}{l}\text { Quality Rating and } \\
\text { Comments }\end{array}$ \\
\hline $\begin{array}{l}\text { Hoggett and } \\
\text { Randall } \\
2018 \\
\text { Country not } \\
\text { reported }\end{array}$ & $\begin{array}{l}\text { In-depth qualitative } \\
\text { interviews } \\
\text { Thematic analysis }\end{array}$ & $\begin{array}{l}\text { To understand how } \\
\text { scientists and activists } \\
\text { psychologically manage } \\
\text { their work on climate } \\
\text { change } \\
\text { To examine what } \\
\text { emotional resilience factors } \\
\text { are present }\end{array}$ & $\begin{array}{l}n=16 \text { (climate scientists } 6, \\
\text { climate activists } 10 \text { ) } \\
\text { Ages not well reported } \\
\text { (activists } 20 \mathrm{~s}-50 \mathrm{~s}, \\
\text { scientists } 1 \text { young, } 5 \text { senior) } \\
\text { Gender not reported }\end{array}$ & $\begin{array}{l}\text { Activists: } \\
\text { 'Crisis' stage of journey } \\
\text { involved urgency, terror, } \\
\text { anger, feeling } \\
\text { overwhelmed, } \\
\text { disempowerment, difficult } \\
\text { to resolve } \\
\text { Anxiety from engagement } \\
\text { with direct action (police, } \\
\text { law) } \\
\text { Burnout/depression in } \\
9 \text { /10 activists } \\
\text { Scientists: } \\
\text { Anxiety from burden of } \\
\text { responsibility } \\
\text { Distress at disagreement } \\
\text { with colleagues, public } \\
\text { perception of science, fear } \\
\text { of speaking out, media } \\
\text { attacks }\end{array}$ & $\begin{array}{l}\text { Activists' } \\
\text { trajectory: epiphany, immersion, } \\
\text { crisis, resolution through sense of } \\
\text { agency, action as antidote to } \\
\text { despair } \\
\text { Activists managing emotional } \\
\text { impacts through positive and } \\
\text { concrete view of the future, } \\
\text { sophisticated and supportive } \\
\text { network of practice, and emphasis } \\
\text { on self-care } \\
\text { Scientists' trajectory: gradual } \\
\text { realisation } \\
\text { More variability in how } \\
\text { knowledge affects private life } \\
\text { Frustration/anger at public } \\
\text { indifference } \\
\text { Scientists managing emotional } \\
\text { impacts through use of } \\
\text { institutional defences: scientific } \\
\text { progress, excitement of the work, } \\
\text { detachment, rationality, } \\
\text { specialisation, overwork }\end{array}$ & $\begin{array}{l}\text { Both parties work directly } \\
\text { with climate change issues } \\
\text { in daily work } \\
\text { Role (activist vs. scientist) } \\
\text { demonstrated significant } \\
\text { differences in trajectory of } \\
\text { emotional impact, } \\
\text { engagement in public } \\
\text { sphere and managing } \\
\text { emotional impacts }\end{array}$ & $\begin{array}{l}\text { B } \\
\text {-No funding source or } \\
\text { consideration of ethics } \\
\text {-Poor description of } \\
\text { demographics of } \\
\text { participants } \\
\text {-Sampling bias } \\
\text {-No data saturation } \\
\text {-Data collection and } \\
\text { recording unclear } \\
\text {-Unclear how many people } \\
\text { analysed the results } \\
\text {-Quotes not matched to } \\
\text { demographics } \\
\text {-Limitations not discussed } \\
\text { in detail } \\
\text {-No reflexivity } \\
\text {-Utilised member checking }\end{array}$ \\
\hline $\begin{array}{l}\text { Howard et al } \\
2020 \\
\text { USA }\end{array}$ & $\begin{array}{l}\text { Mixed method: } \\
\text { quantitative survey with } \\
\text { single open-ended } \\
\text { question on how climate } \\
\text { change was contributing to } \\
\text { levels of mental distress } \\
\text { Quantitative analysis: } \\
\text { descriptive and } \\
\text { correlational statistics, } \\
\text { ANOVA } \\
\text { Qualitative analysis: } \\
\text { content analysis with } \\
\text { coding methodology }\end{array}$ & $\begin{array}{l}\text { Primary: "to examine the } \\
\text { association between } \\
\text { climate change risk } \\
\text { perception and mental } \\
\text { well-being among farmers } \\
\text { and ranchers in Montana" } \\
\text { (p. 88) } \\
\text { Secondary: "to examine } \\
\text { how climate change may } \\
\text { be affecting the mental } \\
\text { well-being of farmers and } \\
\text { ranchers in the state" (p. } \\
\text { 88) }\end{array}$ & $\begin{array}{l}n=125 \\
\text { Gender and race not } \\
\text { recorded but were } \\
\text { predominantly male and } \\
\text { white } \\
\text { Age } 18-34(21.7 \%), 35-54 \\
(49.2 \%), 55+(29.2 \%) \\
\text { Farmers and ranchers in } \\
\text { Montana, USA }\end{array}$ & $\begin{array}{l}\text { Financial concern: worry } \\
\text { about reduced crop yields, } \\
\text { no funds to mitigate } \\
\text { impacts, land and } \\
\text { investments rendered } \\
\text { useless, no one to buy } \\
\text { business, children will not } \\
\text { take over } \\
\text { Operational planning: } \\
\text { worry about } \\
\text { unpredictability of climate } \\
\text { affecting planting, crop } \\
\text { choice }\end{array}$ & $\begin{array}{l}\text { Resilience: changing farming, } \\
\text { action response, have to adapt, be } \\
\text { creative, flexibility, support } \\
\text { politicians/groups helping }\end{array}$ & $\begin{array}{l}\text { Agricultural workers with } \\
\text { livelihoods depending on } \\
\text { land } \\
\text { Affiliation: organic farmers } \\
\text { had significantly greater } \\
\text { anxiety compared to } \\
\text { conventional } \\
\text { Operation focus: fruit/ } \\
\text { vegetable farmers had } \\
\text { significantly greater } \\
\text { anxiety than grain/legume } \\
\text { farmers } \\
\text { Contribution to income: } \\
\text { those with farming } \\
\text { contributing to } 10-70 \% \\
\text { income had significantly } \\
\text { greater anciety compared } \\
\text { to } 70-100 \% \\
\text { No significant differences } \\
\text { in anxiety by age, } \\
\text { generation or years } \\
\text { working in agriculture }\end{array}$ & $\begin{array}{l}\text { A } \\
\text {-Possible sampling bias } \\
\text {-Quotes not matched to } \\
\text { demographic data } \\
\text {-Very small but targeted } \\
\text { qualitative component } \\
\text {-Survey pre-tested for } \\
\text { validity } \\
\text {-Qualitative coding by two } \\
\text { separate authors with high } \\
\text { Cohen's kappa agreement } \\
\text { score }\end{array}$ \\
\hline
\end{tabular}


Table 1. Cont.

\begin{tabular}{|c|c|c|c|c|c|c|c|}
\hline $\begin{array}{c}\text { Author, Year, } \\
\text { Country of Study }\end{array}$ & Design and Methodology & Aim & Participants and Setting & $\begin{array}{l}\text { Anxiety Themes } \\
\text { Identified (Including } \\
\text { Themes about Worry } \\
\text { Where Relevant) }\end{array}$ & Other Themes Identified & $\begin{array}{c}\text { Factors Influencing } \\
\text { Experience of Climate } \\
\text { Change }\end{array}$ & $\begin{array}{l}\text { Quality Rating and } \\
\text { Comments }\end{array}$ \\
\hline $\begin{array}{l}\text { Kemkes and } \\
\text { Akerman } \\
2019 \\
\text { USA }\end{array}$ & $\begin{array}{l}\text { Structured narrative } \\
\text { interviews } \\
\text { Interpretive } \\
\text { phenomenological analysis }\end{array}$ & $\begin{array}{l}\text { Explored participant } \\
\text { understanding of climate } \\
\text { change, worry about } \\
\text { future changes, and who is } \\
\text { responsible for addressing } \\
\text { climate change }\end{array}$ & $\begin{array}{l}n=17 \text { (M 10, F 7) } \\
\text { Ages 36-80 (mean age 56) } \\
\text { Community members } \\
\text { living on shore of Lake } \\
\text { Superior, Wisconsin USA }\end{array}$ & $\begin{array}{l}\text { Themes were not explicitly } \\
\text { identified but extrapolated } \\
\text { from data provided: } \\
\text { Anxiety from failure of } \\
\text { collective action and } \\
\text { futility of individual } \\
\text { change } \\
\text { Uncertainty about local } \\
\text { environmental changes, } \\
\text { fear of major weather } \\
\text { events } \\
\text { Overwhelmed but acting } \\
\text { ethically at an individual } \\
\text { level } \\
\text { "One-word responses" } \\
\text { within the anxiety } \\
\text { spectrum (scared, terrified, } \\
\text { concerning, concerned) }\end{array}$ & $\begin{array}{l}\text { Silence around climate change: } \\
\text { inability to talk of climate change } \\
\text { in certain settings } \\
\text { Issue diluted amongst other } \\
\text { things to be worried about } \\
\text { Other one-word responses } \\
\text { included negative (catastrophe, } \\
\text { apocalyptic, hopeless/helpless, } \\
\text { depressed), positive (hopeful, } \\
\text { optimistic) words, as well as } \\
\text { words related to scale of climate } \\
\text { change (impending, inevitable, } \\
\text { humbled) }\end{array}$ & $\begin{array}{l}\text { Although demographic } \\
\text { characteristics were } \\
\text { gathered, they were not } \\
\text { linked to themes derived } \\
\text { from the data }\end{array}$ & $\begin{array}{l}\text { C } \\
\text {-Sample size and data } \\
\text { saturation not addressed } \\
\text {-Sampling bias } \\
\text {-Themes not well elicited } \\
\text {-No validation of findings } \\
\text {-No discussion of } \\
\text { limitations } \\
\text {-No reflexivity, no } \\
\text { consideration of ethics } \\
\text {-Both authors performed } \\
\text { data analysis } \\
\text { independently }\end{array}$ \\
\hline $\begin{array}{l}\text { Norgaard } \\
2006 \\
\text { Norway }\end{array}$ & $\begin{array}{l}\text { Ethnographic data (field } \\
\text { research), interviews, } \\
\text { media analysis, participant } \\
\text { observation, focus groups } \\
\text { Analytic method not } \\
\text { documented }\end{array}$ & $\begin{array}{l}\text { Aim not clearly expressed } \\
\text { To explore why people } \\
\text { were not more actively } \\
\text { engaged with global } \\
\text { warming, with a focus on } \\
\text { emotion and emotion } \\
\text { management }\end{array}$ & $\begin{array}{l}n=46(\mathrm{M} 25, \mathrm{~F} 21) \\
\text { Ages }<20(7), 20-35 \text { (8), } \\
\text { 35-60 (19), 69+ (11) Total } 45 \\
\text { Range "19 to early } 70 \mathrm{s"} \\
\text { Residents in a rural } \\
\text { Norwegian community }\end{array}$ & $\begin{array}{l}\text { Fear related to loss of } \\
\text { ontological security } \\
\text { Fear of "being a bad } \\
\text { person", which was a } \\
\text { threat to individual and } \\
\text { national self-concept } \\
\text { Unpleasant emotions } \\
\text { (including fear) managed } \\
\text { through selective attention } \\
\text { (controlling exposure to } \\
\text { information, not thinking } \\
\text { too far ahead, focusing on } \\
\text { something individual can } \\
\text { do) which led to } \\
\text { "movement } \\
\text { non-participation" }\end{array}$ & $\begin{array}{l}\text { Helplessness } \\
\text { Need to maintain optimism, } \\
\text { stoicism } \\
\text { Observed changes over a lifetime } \\
\text { Guilt of contributing to the } \\
\text { problem } \\
\text { Guilt and threats to identity } \\
\text { managed by perspectival } \\
\text { selectivity }\end{array}$ & $\begin{array}{l}\text { Salience and visibility of } \\
\text { climate change due to local } \\
\text { changes } \\
\text { Norway is a wealthy } \\
\text { nation which benefits from } \\
\text { oil production } \\
\text { Occupation: activists and } \\
\text { educators limit access to } \\
\text { information to avoid being } \\
\text { overwhelmed and enable } \\
\text { them to continue their } \\
\text { work }\end{array}$ & $\begin{array}{l}\text { D } \\
\text {-Unclear aims and title } \\
\text {-Sampling not described } \\
\text {-No data saturation } \\
\text {-Poor description of data } \\
\text { recording } \\
\text {-No qualitative analysis } \\
\text { documented } \\
\text {-No respondent validation } \\
\text { or independent data } \\
\text { analysis } \\
\text {-No consideration of } \\
\text { limitations } \\
\text {-Limited reflexivity, } \\
\text { significant subjectivity } \\
\text {-No use of empirical data } \\
\text { to support claims } \\
\text {-Funding source unclear } \\
\text {-Issues with sampling, } \\
\text { analysis, reflexivity, and } \\
\text { ethics threaten overall } \\
\text { validity }\end{array}$ \\
\hline
\end{tabular}


Table 1. Cont.

\begin{tabular}{|c|c|c|c|c|c|c|c|}
\hline $\begin{array}{c}\text { Author, Year, } \\
\text { Country of Study }\end{array}$ & Design and Methodology & Aim & Participants and Setting & $\begin{array}{l}\text { Anxiety Themes } \\
\text { Identified (Including } \\
\text { Themes about Worry } \\
\text { Where Relevant) }\end{array}$ & Other Themes Identified & $\begin{array}{l}\text { Factors Influencing } \\
\text { Experience of Climate } \\
\text { Change }\end{array}$ & $\begin{array}{l}\text { Quality Rating and } \\
\text { Comments }\end{array}$ \\
\hline $\begin{array}{l}\text { Nyantakyi- } \\
\text { Frimpong and } \\
\text { Bezner-Kerr } \\
\text { 2015 } \\
\text { Ghana }\end{array}$ & $\begin{array}{l}\text { Ethnographic fieldwork } \\
\text { guided by feminist } \\
\text { political ecology theory } \\
\text { Multimethod triangulation } \\
\text { of focus grous, individual } \\
\text { interviews, participant } \\
\text { observation, } \\
\text { meteorological data, } \\
\text { household surveys } \\
\text { Quantitative analysis: } \\
\text { descriptive statistics (two } \\
\text { sample test of proportions } \\
\text { in SPSS version 21) } \\
\text { Qualitative analysis: hand } \\
\text { coding and analysis for } \\
\text { themes; participatory } \\
\text { ranking and scoring data } \\
\text { analysed using a method } \\
\text { by Tschakert to calculate } \\
\text { incidence, severity and } \\
\text { importance indices for } \\
\text { each factor }\end{array}$ & $\begin{array}{l}\text { "To explore the relative } \\
\text { importance of climate } \\
\text { change in the context of } \\
\text { multiples stressors in } \\
\text { semi-arid Ghana" (p. 1) } \\
\text { "(i) What factors do } \\
\text { farmers identify as most } \\
\text { relevant for climate change } \\
\text { resilience and adaptation, } \\
\text { and how do these factors } \\
\text { differ by gender, age and } \\
\text { kinship relations? } \\
\text { (ii) how important is } \\
\text { climate change as } \\
\text { compared to other factors } \\
\text { that shape smallholder } \\
\text { farming and food } \\
\text { security?" (p. 40) }\end{array}$ & $\begin{array}{l}n=135 \\
8 \text { Focus groups, } n=75 \\
\text { (young M 19, young F 21, } \\
\text { elderly M 18, elderly F 17) } \\
\text { In-depth interviews } n=60 \\
\text { (M 26, F 34) } \\
6 \text { were key informants } \\
\text { (3 agricultural extension } \\
\text { officers, } 1 \text { NGO worker, } \\
1 \text { nutritionist, and 1 health } \\
\text { surveillance assistant). } \\
\text { All were residents in } \\
2 \text { villages in northwest } \\
\text { Ghana }\end{array}$ & $\begin{array}{l}\text { Farmers are aware but do } \\
\text { not worry about climate } \\
\text { variability and change, } \\
\text { compared to other } \\
\text { concerns } \\
\text { Farmers are used to } \\
\text { extreme weather events } \\
\text { and report that they } \\
\text { already manage risk with } \\
\text { adaptable farming systems } \\
\text { and are used to innovating }\end{array}$ & $\begin{array}{l}\text { Farmers perceive a change in } \\
\text { climate accurately } \\
\text { Concerns vary with gender } \\
\text { - men worry about local weather } \\
\text { events, food prices } \\
\text {-women's greatest concern is } \\
\text { access to household granaries and } \\
\text { labour constraints } \\
\text { Land appropriation was a concern } \\
\text { for all }\end{array}$ & $\begin{array}{l}\text { Significant vulnerability } \\
\text { (poverty, main economic } \\
\text { activities are agriculture } \\
\text { and pastoralism); } 39 \% \\
\text { households are food } \\
\text { insecure } \\
\text { Respondents perceived } \\
\text { themselves as less } \\
\text { vulnerable to climate } \\
\text { change because they } \\
\text { already have adaptive } \\
\text { capacity }\end{array}$ & $\begin{array}{l}\text { A } \\
\text {-No discussion of } \\
\text { limitations, little reflexivity, } \\
\text { ethics not acknowledged } \\
\text {-Did not frame as climate } \\
\text { change research to } \\
\text { participants } \\
\text {-Data saturation reached } \\
\text {-Comprehensive } \\
\text { methodology, data } \\
\text { triangulation } \\
\text {-Survey instrument was } \\
\text { pre-tested } \\
\text {-Findings were validated in } \\
\text { feedback workshops }\end{array}$ \\
\hline
\end{tabular}


Table 1. Cont.

\begin{tabular}{|c|c|c|c|c|c|c|c|}
\hline $\begin{array}{c}\text { Author, Year, } \\
\text { Country of Study }\end{array}$ & Design and Methodology & Aim & Participants and Setting & $\begin{array}{l}\text { Anxiety Themes } \\
\text { Identified (Including } \\
\text { Themes about Worry } \\
\text { Where Relevant) }\end{array}$ & Other Themes Identified & $\begin{array}{l}\text { Factors Influencing } \\
\text { Experience of Climate } \\
\text { Change }\end{array}$ & $\begin{array}{l}\text { Quality Rating and } \\
\text { Comments }\end{array}$ \\
\hline $\begin{array}{l}\text { Petheram et al. } \\
2010 \\
\text { Australia }\end{array}$ & $\begin{array}{l}\text { Fieldwork visits } \\
\text { Semi-structured interviews } \\
\text { (individual and group), } \\
\text { workshops (rich picture } \\
\text { diagramming, } \\
\text { participatory sculpting, } \\
\text { participant-generated } \\
\text { photography) } \\
\text { Constructionist/grounded } \\
\text { theory-based continuous } \\
\text { data gathering and } \\
\text { analysis, analysis of data } \\
\text { with substantive and } \\
\text { theoretical coding }\end{array}$ & $\begin{array}{l}\text { To understand factors } \\
\text { influencing general } \\
\text { vulnerability and adaptive } \\
\text { capacity in the context of } \\
\text { poverty and climate } \\
\text { change in Yolgnu people in } \\
\text { NE Arnhem Land, } \\
\text { Northern Territory }\end{array}$ & $\begin{array}{l}n=21 \text { (M 9, F 12) } \\
\text { Range of older and } \\
\text { younger adults (ages not } \\
\text { specified) } \\
\text { Community members in } \\
\text { East Arnhem Land (male } \\
\text { indigenous land/sea } \\
\text { rangers and women from } \\
\text { local households and a } \\
\text { women's organisation) }\end{array}$ & $\begin{array}{l}\text { Climate change was less of } \\
\text { a concern than other issues } \\
\text { affecting the community } \\
\text { Climate change will } \\
\text { exacerbate existing } \\
\text { concerns, cannot be } \\
\text { considered in isolation } \\
\text { from non-climate issues } \\
\text { Raising awareness of } \\
\text { climate change so school } \\
\text { children do not worry }\end{array}$ & $\begin{array}{l}\text { Participants were unclear about } \\
\text { Western notions of climate change } \\
\text { Differences in world view and } \\
\text { miscommunication between } \\
\text { participants and "Balanda" (white } \\
\text { Australians) } \\
\text { Preferences for adaptation } \\
\text { strategies included sustainability } \\
\text { and greater value on traditional } \\
\text { and cultural practices } \\
\text { Specific changes in landscape had } \\
\text { been noted in recent years and } \\
\text { caused distress } \\
\text {-Taking care of country } \\
\text {-Concern about current and future } \\
\text { situation of communities and } \\
\text { wanting change to relieve poverty } \\
\text { and other worrying issues }\end{array}$ & $\begin{array}{l}\text { Population highly } \\
\text { vulnerable to climate } \\
\text { change (poverty, lack of } \\
\text { agency and adaptive } \\
\text { capacity, historic events, } \\
\text { multiple pressing } \\
\text { biopsychosocial issues) } \\
\text { Indigenous population } \\
\text { with strong connection to } \\
\text { place and sensitivity to the } \\
\text { natural landscape }\end{array}$ & $\begin{array}{l}\text { A } \\
\text {-No funding source } \\
\text { nominated } \\
\text {-Adapted research aims } \\
\text { based on findings to be of } \\
\text { more utility/relevance } \\
\text {-Data saturation reached } \\
\text {-Participant verification } \\
\text { occurred } \\
\text {-Used culturally adapted } \\
\text { methods to gather data } \\
\text {-Good reflexivity }\end{array}$ \\
\hline $\begin{array}{l}\text { Ryghaug et al. } \\
2011 \\
\text { Norway }\end{array}$ & $\begin{array}{l}\text { Focus group interviews } \\
\text { Analysis 'inspired' by } \\
\text { grounded theory; } \\
\text { domestication theory used } \\
\text { as a basis for making story } \\
\text { lines }\end{array}$ & $\begin{array}{l}\text { To analyse how people } \\
\text { reason about and make } \\
\text { sense of human-made } \\
\text { global warming, in light of } \\
\text { two previously identified } \\
\text { categories of media } \\
\text { representations, the } \\
\text { "nature drama" and } \\
\text { "science drama" }\end{array}$ & $\begin{array}{l}n=62 \text { in } 10 \text { focus groups } \\
\text { (M 24, F 38) } \\
\text { Age range } 16-71 \\
\text { Community members in } \\
\text { Norway } \\
\text { Focus groups were existing } \\
\text { social networks } \\
26 / 62(42 \%) \text { were students }\end{array}$ & $\begin{array}{l}\text { Climate change as a } \\
\text { frightening scenario } \\
\text { Worry for future } \\
\text { generations was linked to } \\
\text { extreme weather striking } \\
\text { climate incidents ("nature } \\
\text { drama") } \\
\text { Worry not pervasive, as } \\
\text { frightening events are not } \\
\text { happening here (their } \\
\text { country) or now }\end{array}$ & $\begin{array}{l}\text { Scientific controversy } \\
\text { Role of the media as the main } \\
\text { source of information about } \\
\text { climate change, belief that media } \\
\text { overemphasises threat } \\
\text { Four ways of domesticating } \\
\text { knowledge: acceptors, tempered } \\
\text { acceptors, uncertain and sceptics }\end{array}$ & $\begin{array}{l}\text { Participants from wealthy } \\
\text { stable country } \\
\text { Climate change perceived } \\
\text { as distant from everyday } \\
\text { concerns, less imminent } \\
\text { than other problems }\end{array}$ & $\begin{array}{l}\text { C } \\
\text {-Bias in sampling } \\
\text {-No comment on data } \\
\text { saturation } \\
\text {-No respondent validation } \\
\text {-No discussion of } \\
\text { limitations } \\
\text {-No reflexivity, which is } \\
\text { especially important given } \\
\text { how subjects were } \\
\text { recruited and inherent bias } \\
\text {-Ethical flaws }\end{array}$ \\
\hline
\end{tabular}


Table 1. Cont.

\begin{tabular}{|c|c|c|c|c|c|c|c|}
\hline $\begin{array}{c}\text { Author, Year, } \\
\text { Country of Study }\end{array}$ & Design and Methodology & Aim & Participants and Setting & $\begin{array}{l}\text { Anxiety Themes } \\
\text { Identified (Including } \\
\text { Themes about Worry } \\
\text { Where Relevant) }\end{array}$ & Other Themes Identified & $\begin{array}{l}\text { Factors Influencing } \\
\text { Experience of Climate } \\
\text { Change }\end{array}$ & $\begin{array}{l}\text { Quality Rating and } \\
\text { Comments }\end{array}$ \\
\hline $\begin{array}{l}\text { Wright and Nyberg } \\
2012 \\
\text { Australia }\end{array}$ & $\begin{array}{l}\text { Individual semi-structured } \\
\text { interviews and analysis of } \\
\text { documents (strategy docs, } \\
\text { communication material, } \\
\text { submissions to gov't, } \\
\text { media coverage) } \\
\text { Abductive approach } \\
\text { Coding of emotional } \\
\text { expressions using QSR } \\
\text { NVivo software }\end{array}$ & $\begin{array}{l}\text { (i) To explore how } \\
\text { organisations have } \\
\text { responded to the } \\
\text { evidenced emotionality of } \\
\text { climate change in their } \\
\text { corporate environmental } \\
\text { practices } \\
\text { (ii) To explore how } \\
\text { sustainability specialists } \\
\text { manage their own } \\
\text { emotions in the process of } \\
\text { emotionology work }\end{array}$ & $\begin{array}{l}n=36 \text { (M 21, F 15) } \\
\text { Age range 25-60 } \\
\text { Sustainability specialists in } \\
\text { corporate industries in } \\
\text { Australia }\end{array}$ & $\begin{array}{l}\text { "Climate change as threat" } \\
\text { (anxiety and apprehension } \\
\text { in regard to the future } \\
\text { implications for society } \\
\text { and the economy) } \\
\text { Anxiety was harnessed to } \\
\text { improve employee } \\
\text { engagement, productivity, } \\
\text { and corporate reputation } \\
\text { Sustainability } \\
\text { managers/consultants } \\
\text { downplay threat and } \\
\text { promote } \\
\text { challenge/opportunity } \\
\text { linked to business concerns } \\
\text { Managing emotions } \\
\text { through calculative } \\
\text { methods, constraining, } \\
\text { championing and } \\
\text { compartmentalising } \\
\text { emotions }\end{array}$ & $\begin{array}{l}\text { "Climate change as battleground } \\
\text { or conflict" } \\
\text { (frustration, anger, and hostility) } \\
\text { and "climate change as challenge } \\
\text { and opportunity" (hope, } \\
\text { enthusiasm, and excitement) } \\
\text { Themes regarding processes of } \\
\text { emotionology work (spanning, } \\
\text { changing, or creating } \\
\text { emotionologies) }\end{array}$ & $\begin{array}{l}\text { Sustainability specialists } \\
\text { working directly with } \\
\text { climate change issues, all } \\
\text { believed in climate change } \\
\text { and were passionate } \\
\text { Age, type of organisation } \\
\text { and gender were not } \\
\text { discussed as qualifiers }\end{array}$ & $\begin{array}{l}\text { C } \\
\text {-Poor presentation of data } \\
\text { findings } \\
\text {-No reflexivity } \\
\text {-No comment on data } \\
\text { saturation } \\
\text {-Unclear how data from } \\
\text { documents were gathered } \\
\text { and analysed } \\
\text {-Sampling method not } \\
\text { described } \\
\text {-No validation of findings } \\
\text {-No dissenting views } \\
\text {-Ethical flaws }\end{array}$ \\
\hline
\end{tabular}




\subsection{Thematic Analysis}

The results of the systematic review were divided into two broad areas of understanding about climate change anxiety; namely, (i) the "scope of climate change anxiety", i.e., what was the specific reason for or focus of anxiety, and (ii) "responses to climate change anxiety", which included emotional responses and ways of coping with anxiety (Table 1). Multiple themes emerged for each and are presented below. Results for the secondary aim, to explore the factors influencing climate change anxiety, are also provided in Table 1.

\subsubsection{Themes Related to the Scope of Climate Change Anxiety}

A number of key themes emerged regarding the focus of climate change anxiety, which included worry about threats to livelihood, worry for future generations, worry about apocalyptic futures, anxiety at the perceived lack of response to climate change, and competing worries.

Worry about Threats to Livelihood

Disruptions to livelihood were a source of anxiety in many studies $[24,26,27,29,30,36]$. Threats to daily life identified by participants included adverse economic changes, migration, extreme weather events, and lack of disaster preparedness. Many feared the loss of access to resources resulting in water scarcity, disruptions to food supplies and crop production, and impacts on local agriculture and the economy. Villagers in Fiji were concerned that:

"Maybe in the future our plants can't grow and we have to go buy them from the market" [36] (p. 105)

Water scarcity was a concern in a lakeside community in Canada:

"You need water, like you can't survive without that so I do worry about ... access to clean water" [26] (p. 78)

Financial worries were the most prominent for the predominantly male farmers interviewed in America:

"as with most, my worries generally stem from financial stress" [29] (p. 91)

Several studies highlighted concern about climate change-related migration. This included people who anticipate being forced to migrate:

"I know I'll be leaving soon, but when news comes that Tuvalu is affected or will sink, it makes me cry. Because I was born here, I'm Tuvaluan" [27] (p. 4)

In contrast, in countries less immediately threatened by climate change, such as the United Kingdom, the worry related to how to accommodate incoming climate refugees [36].

The increased frequency of extreme weather events and their links to livelihoods was a concern in Wisconsin:

"I'm afraid we're really gonna start getting hit with like massive tornadoes. That's my biggest fear" [30] (p. 6)

There were divergent subthemes of "opportunity" and "already adapted". Some perceived new livelihood opportunities related to the changing climate. A respondent in the fishing village of Kodiak, Alaska, was optimistic:

"A disaster for some may mean prosperity for others - as polar ice melts, we might benefit as more shipping traffic comes through this area" [24] (p. 292)

The business opportunities presented by the challenges of climate change were emphasised by sustainability consultants in corporations in Australia:

"Answering the challenge and being part of the climate change solution can have a multitude of immediate and long-term benefits for business" [35] (p. 1572) 
Some people did not perceive threats to their way of life, as they had "already adapted". For example, among farmers in Ghana, there was a degree of confidence about adapting to environmental change, which seemed to mitigate against anxiety:

"Let's face it, people have already seen extreme weather events in the past. Very bad ones. So they keep finding innovative ways of dealing with the weather changes. That's why we are aware but don't generally worry about environmental changes" [32] (p. 52)

Worry for Future Generations

Worry about future generations was a prominent theme in more developed countries $[24,26,29,34,36]$. The uncertainty of what sort of world would be left for children and grandchildren was a source of distress. For example, a participant in Ontario, Canada, stated:

"We are actually taking our planet on a crash course and as a grandmother, I am deeply, deeply, deeply concerned about this. I have a one-year-old granddaughter now. You know we are leaving nothing for her" [26] (p. 80)

A divergent subtheme regarding worry for future generations was that of hope. Future generations were seen as a source of hope and potential solutions amongst young people in South Korea:

"Hope may come from education" [23] (p. 67)

Worry about Apocalyptic Futures

For some, climate change was a "frightening scenario" [34] (p. 784) that evoked anxiety. "Catastrophe" and "apocalypse" were amongst the single words chosen to describe how participants felt about climate change in an American study [30] (p. 4). Students in South Korea associated climate change with doomsday scenarios they had seen in the media:

"Something like this will happen to humans; we cannot prevent destroying the earth ... we will die" [23] (p. 68)

Anxiety at the Perceived Lack of Response to Climate Change

The failure of others to take action was a source of anxiety. Participants felt that climate change could only be talked about in certain groups, which increased their anxiety $[23,30,35]$, and not everyone shared the participants' worries:

"The first thing I associate when I hear climate change is terrified, you know. People don't think it exists, or it's happening, or nobody is going to do anything about it" [30] (p. 5)

Gaining Perspective-Competing Worries

Some participants struggled to place climate change amongst multiple other more pressing concerns in their daily life [30,32-34]. This was true in Western cultures, such as Wisconsin, USA:

"There's so much to be worried about, it's diluted you know, what we have time to talk about" [30] (p. 5)

However, the awareness of competing worries was particularly true of participants in areas where resources were already scarce. Grape growers in Australia identified multiple financial and political stressors that they placed ahead of climate change:

"Climate change is the least of my worries" [25] (p. 6)

Amongst Yolgnu people in Arnhem Land, Australia, local social and ecological issues, such as mining and observed seasonal changes, were highlighted as more immediate concerns than anthropogenic climate change which, in fact, was barely mentioned [33]. Climate change was not viewed as a separate entity from other worries, but was perceived as interwoven and likely to exacerbate existing worries, which, as in Ghana [32], were deemed to be much more important than climate change. 


\subsubsection{Themes in Responses to Climate Change Anxiety}

A spectrum of responses to climate change anxiety emerged, including symptoms of anxiety, the feelings of helplessness and disempowerment that climate change anxiety evoked, and finally a complex theme encompassing the ways of managing climate change anxiety. Distancing and avoidance, taking action, fostering support, adapting, and actively choosing optimism and hope were themes regarding strategies employed to manage climate change anxiety.

\section{Symptoms of Anxiety}

Participants described worry about climate change in ways that were consistent with symptoms of clinical anxiety. This included feelings of suffocation [23], panic amongst climate change activists [28], and rumination on negative emotions of guilt and worry [37]. In Tuvalu, residents described disturbed sleep, poor concentration, inability to relax, and other effects on function related to anxiety about climate change:

"Sometimes I want to sleep, but I can't because those thoughts about climate change keep popping up ... thoughts about this distract me from my study" [27] (p. 4)

Feeling Helpless and Disempowered

People across Europe [34,36], North America [24,30], Australia [25], and in Tuvalu [27] described a sense of powerlessness and feeling overwhelmed. They did not know what to do to tackle the problem of climate change, feeling individual measures were futile, which led to feelings of depression and anxiety:

"I feel that you resign a little, this is too big. This makes you feel like: Help, what can you do other than trouble yourself?" [34] (p. 785)

Disempowerment was a strong theme amongst South Australian grape growers, where there was distrust of climate change information because:

"some of the information is too extreme, so if those projections happen we're finished anyway" [25] (p. 7)

\section{Managing Climate Change Anxiety}

Five themes emerged describing strategies to manage climate change anxiety.

a. Distancing and avoidance

A key theme which emerged as a way to manage climate change anxiety was distancing and avoidance. Some saw climate change as something geographically and temporally distant, which was protective against anxiety [24]. Some downplayed the seriousness of climate change to alleviate worry, such as this university student in Norway:

"I do not think it [global warming] is as human-made ... as it allegedly is, according to the tabloids ... I do not think it is as bad as they want to describe it" [34] (p. 789)

Even those who engage with climate change on a daily basis in their work made conscious decisions to distance themselves from climate change anxiety. For activists, this was a key step in resolving feelings of crisis brought on by knowledge of climate change:

"I barely think about climate change now. It's in the background of my life all the time, but I rarely sit and actually talk about climate change or read very much about it" [28] (p. 230)

Climate scientists and sustainability managers both emphasised rationality in their daily work, managing emotions by restricting or compartmentalising. However, this approach to coping related to emotions in general and not anxiety specifically:

"I think a lot of scientists convey the impression that they have no feelings at all about these issues" [28] (p. 236) 


\section{b. Taking action}

A move towards action was another strategy that was used to manage worry about climate change. Young people in Sweden took actions, such as researching solutions and modifying behaviour, to cope with climate change anxiety [37]. Amongst farmers in the USA, "taking action" was linked to a sense of resilience:

"Action, not worry, solves the problem" [29] (p. 91)

This sentiment was echoed by climate activists who developed a sense of agency that allowed them to move forward:

"Action is the antidote to despair" [28] (p. 229)

c. Fostering support

Actively seeking support was an important theme that emerged in young people [37] and in the specific population of climate change activists who were dealing with overwhelming feelings about climate change [28]. The activists consciously held positive ideas about the future and identified the importance of a network of practice and culture of self-care:

"We build into it after the event, doing something where we talk about the emotions of how to deal with that" [28] (p. 234)

d. Adapting

Developing confidence in the ability to adapt to climate change appeared to be linked to a sense of resilience. This was seen in general community members in America:

"There are so many things we don't know. We adapt. We've always adapted" [24] (p. 291)

Confidence about adaptation was particularly found in groups of farmers in the USA [29] and in Ghana [32]. These farmers appeared to be accustomed to adaptation, and this flexibility allowed them to deal with depressed and anxious feelings:

"I see the changes and how they are affecting my farm but I am changing how I am farming in response to climate change rather than being depressed by it" [29] (p. 91)

e. Optimism and hope

Actively choosing to be optimistic and hopeful were responses used to manage climate change anxiety $[23,24,26,35,37]$. Focus on positive emotions was notable amongst young people in Sweden [37]. Positive reappraisals of the problem, positive thinking, and fostering existential hope were subthemes identified in the Swedish youth:

"You have to feel hope to make things any better. If no one felt hope then you might as well give up" [37] (p. 547)

Focus on solutions and trust in science, policy, and environmental groups, amongst others, also helped young people cope with anxiety:

"Because a lot of people are working, planting new trees, dealing with the waste and exhaust fumes from cars" [37] (p. 549)

Hope was also harnessed by sustainability managers in their daily work in Australian corporate environments:

"I guess I've always been a bit of an optimist and you have to be in this game. I've got a hope in terms of human ingenuity that we all trade out of this somehow" [35] (p. 1569)

\subsubsection{The Intersection of Anxiety, Sadness, and Solastalgia}

Parallel to climate change anxiety, people described feelings of sadness and loss that were difficult to separate from their experiences of worry $[23,24,27,30,36]$. They repeatedly referred to local changes observed in their environment, which were seen as personal experiences of climate change. A loss of culture and community was a source of both anxiety and sadness in Fiji: 
"Seeing the changes makes me feel sad because people are not engaged ... in helping protect the village and community" [36] (p. 105)

Changes in landscapes and seasons were referred to with a sense of loss in Korea:

"Now I do not feel spring or fall weather as much now...I feel like I lost something" [23] (p. 68)

The emotional impact of local environmental change was strongly felt amongst Indigenous Yolgnu people in Arnhem Land, Australia, with their unique connection to the land:

"What we are doing to mother nature. Mother nature is now weeping" [33] (p. 686)

\section{Discussion}

The aims of this systematic review were to qualitatively explore the scope of climate change anxiety as a concept and to investigate the spectrum of anxiety responses to climate change. A secondary aim was to explore the factors influencing climate change anxiety. This systematic review of the qualitative literature contributes to our understanding of this important, understudied topic. A qualitative approach is best suited to develop an in-depth understanding of new concepts, such as climate change anxiety [20]).

The scope of anxiety about climate change was broad. The majority of studies explored aspects of climate change worry, a component of climate change anxiety. The review identified that "worry about threats to livelihood" was a major concern across all populations, with a breadth of focus from anxiety about access to food and water, to anxiety about finances, population movement, and natural disasters. It is surprising that no known previous study has explored the specific focus of climate change anxiety in a quantitative manner, despite numerous studies measuring the prevalence of climate change anxiety $[19,38-46]$, and studies assessing the scope of environmental worries beyond climate change [13,47-49]. The theme of "worry about future generations" has been identified previously [50], as have correlations between parenthood and anxiety about climate change [41]. In this study, "worry about future generations" was more prominent amongst Western countries [24,26,29,34,36], where participants tended to perceive climate change as a future rather than current threat. Anxiety about "apocalyptic futures" is likely to be found in those with egalitarian beliefs, who have higher perceptions of catastrophe related to climate change [38].

The systematic review revealed patterns in the demographic characteristics of participants and climate change anxiety, addressing the secondary aim of this study. The key factors influencing emergent themes for the scope of climate change anxiety were vulnerability to climate change, socioeconomic factors, and occupation (Table 1). Four of the twelve studies included participants from either developing countries (Fiji, Ghana, Tuvalu) or disadvantaged socioeconomic groups (Arnhem Land, Australia). These participants are arguably amongst the most vulnerable to the effects of climate change [1]. Despite their high vulnerability, two of these studies identified a relative lack of anxiety or worry about climate change [32,33] when compared to more developed nations in North America and Europe [24,26,30,34]. There are several potential explanations for this finding. In Arnhem Land, it was noted that Western concepts of climate change were very poorly understood [33]. Further, more immediate concerns about day-to-day survival were prominent there and in Ghana, giving rise to the theme of "competing worries". Amongst populations already struggling to survive on the land, climate change can be seen as "yet another stressor" [51] (p. 1). In Tuvalu, by contrast, knowledge of climate change is strong, exemplified by the commonly known trope that "Tuvalu is sinking" [27] (p. 2). Consistent with this, very high levels of climate change anxiety were found in Tuvaluan residents [27], reflecting the level of threat that climate change poses to this island nation [52].

Occupation emerged as a likely influence upon the scope of anxiety about climate change. In North America, farmers (in a predominantly white male participant group) identified financial concerns about the impact of climate change [29]. Grape growing 
farmers in Australia showed a comparative lack of worry about climate change, as they were more concerned with perceived immediate stressors, such as farming costs and viability, alongside scepticism about the existence or seriousness of climate change [25]. Other farming populations have been demonstrated to hold higher rates of scepticism, with associated lower rates of worry about climate change, than the general public $[43,53]$. The present review also found that farmers in Ghana have little anxiety about climate change, as they are confident in their ability to adapt [32].

A gender-related difference in the scope of anxiety might be expected, given that women often experience higher levels of climate change anxiety than men [54-56]. However, the impact of gender on the scope of anxiety was not well explored by the reviewed studies. Only one study noted that women were more likely to worry about future generations, while men worried more about finances [24]. It was not possible to identify any patterns relating to age and the scope of anxiety, as the only paper that specifically studied young people assessed only responses to climate change anxiety, not the scope of anxiety [50]. Given the disproportionate impact of climate change on young people, further study in this population is needed.

The second group of themes that emerged in this review were the responses to climate change anxiety, which ranged from "symptoms of anxiety", to "feeling helpless and disempowered", and "managing climate change anxiety: distancing and avoidance", "taking action", "fostering support", "adapting", and "optimism and hope". The proximity to the effects of climate change corresponded with anxiety responses. "Symptoms of anxiety", and the subsequent impact on daily function, were described by Tuvaluan citizens [27], who arguably face the most tangible threat from climate change. Similarly, the proximity of climate change activists to the realities of climate change threats precipitated feelings of panic [28], whereas such intense experiences of anxiety were less evident in other populations studied within this review. Feeling "helpless and disempowered", however, in the face of anxiety about climate change, was a phenomenon commonly expressed across diverse geographical locations $[24,25,27,28,30,34,36]$. It is likely that this emotion is influenced by individual ontological belief systems, which have been shown to mediate feelings of helplessness in association with climate change anxiety [38].

This review did not reveal any clear factors influencing the particular ways of managing climate change anxiety. Compared to factors influencing the scope of climate change anxiety, it is likely that responses to anxiety (including worry specifically) are less influenced by characteristics, such as gender, location, or social group, but by individual psychological factors and ontological beliefs [39,40,57]. Further, the significant heterogeneity of climate change understanding, perception, and engagement in populations across the world is an ongoing challenge for research [54,57]. "Fostering support" and "distancing and avoidance" have small but significant effects on lessening the experience of psychological distress related to climate change [58]. Among 12-year-olds and adolescents, higher levels of worry were found in those who used "problem-focused coping", where one focuses on ways to solve the problem, such as searching for information [50,59]. It was suggested that 12-year-olds who emphasised the positive affects of optimism and hope may be buffered against negative affects, including worry about climate change [59]. The importance of hope is increasingly recognised within the climate change literature as a part of psychological adaptation and as a way of overcoming difficult emotions associated with knowledge of climate change $[60,61]$.

\subsection{Solastalgia and Sadness}

In this review, solastalgia and sadness emerged as key affects linked to climate change anxiety. Whilst quantitative results demonstrate that anxiety and sadness are frequently the two most common emotional responses to climate change [24,27], the qualitative approach of this paper highlights how closely these emotional states are interconnected, just as in clinical populations [62]. The experience of solastalgia and its relevance amongst Indigenous peoples is particularly recognised $[7,63,64]$ and was also found in this study [33]. 


\subsection{Clinical Implications}

This review highlights the spectrum of anxiety people may experience in relation to climate change, and their responses to those anxieties. Worry, a cognitive component of climate change anxiety, was most commonly explored in these studies. Clinical presentations related to climate change anxiety have not been well elucidated, although they have been recognised in psychotherapeutic spaces [65] and at an obsessive compulsive disorder treatment clinic [66]. The treatment of eco-anxiety, a closely related concept, is an emerging area of research [67]. Evidence of clinical symptoms of anxiety emerged as a small but important theme in this review of the qualitative literature. Quantitative studies in this area report mixed results $[19,46,68-70]$, but convincing evidence of a link between climate change anxiety and clinical anxiety is currently lacking. A survey of primary care patients in America identified a link between climate change concern and dysphoria, but no link to anxiety [70]. Another survey of Australian university students and community members found a small association between climate change distress and depression, and a moderate correlation between climate change distress and future worry [19]. Perceived ecological stress predicted depressive symptoms in a survey of parents in America [69]. None of these studies could demonstrate causation in either direction. Other studies have found no significant correlation between worry about climate change or ecology and psychiatric morbidity from anxiety $[46,68]$.

An alternative view is that climate change anxiety is not a clinical entity, but rather an existential one [11]. The argument for understanding climate change anxiety as an existential worry, rather than a pathological worry of the kind recognised by psychiatry, is reflected by the fact that only two of the fifteen papers were published in medical or psychological journals [27,29]; the remainder appeared in environmental or sociological journals. Further, concern exists about pathologizing climate change anxiety, which has been described as a proportionate emotional response to the current environmental situation $[12,60]$. However, the descriptions of anxiety symptoms, associated distress, and impacts on behaviours and functioning that emerged from this qualitative review should not be ignored.

Further research is required to identify people who are vulnerable to experiencing climate change anxiety at a level of clinical significance and who may benefit from intervention. Validated screening tools, such as the Environmental Distress Scale [71] or the scale developed by Clayton and Karazsia [13], may be helpful. People who screen positive for climate change anxiety may benefit from intervention. However, there is a dearth of literature guiding approaches to its management [67]. A recent scoping review highlighted emerging approaches to treating eco-anxiety, including both individual and group work, predominantly underpinned by ecotherapy, psychoanalysis, and Jungian depth psychology [67]. Notably, only two of the reviewed studies empirically evaluated their psychotherapeutic approach, emphasizing the need for more study [67].

Not all studies of the perceptions of climate change have identified anxiety within the spectrum of responses [25,32,42]. Further study is required to determine the reasons behind this, be they methodological or cohort based. Other emotional responses to climate change, such as anger, guilt, and hope, are likely to have their own patterns and associations [72] and require further study to determine their clinical significance and how they intersect with climate change anxiety.

\subsection{Strengths and Limitations of the Included Papers}

Few of the papers explored participants' knowledge and understanding of climate change, which may affect the reliability of results-why worry about something that you do not believe exists? In the two papers that did measure belief in climate change, it was as low as $58 \%$ in one study population [25], and showed significant variation (55-88\%) between subsets of participants in another [24]. Another study included expressions of scepticism about the seriousness of the climate change threat [34]. In the study by Petheram and colleagues, it became clear to researchers that there were significant misconceptions 
about notions of climate change, and participants' worry increased as they were educated over the course of the study [33]. Multiple other studies have highlighted the poor knowledge and understanding of climate change concepts [44], particularly in the developing world [73-76]. Knowledge of climate change can also be mediated by factors, such as media exposure, which is positively related to the experience of climate change anxiety [77], though its influence was not captured in any of the papers in this review.

The quality of the included studies must be considered. One third of the studies were rated A (no or few flaws), one third were rated as B (containing some flaws), and four of the fifteen were rated $C$ (considerable flaws but still of some value). More weight should be placed on the results from studies of higher quality. Reflexivity and ethics were particularly poorly addressed by the studies, perhaps because many were conducted within ethnographic rather than clinical research frameworks. Additionally, several studies were limited by inadequate sampling $[23,28,30,34,35]$ and a limited application of techniques for validating results $[23,24,26,30,34-37]$.

\subsection{Strengths and Limitations of the Review}

Strengths of this review include a thorough literature search intended to capture any study that included qualitative data on climate change anxiety (including worry), even where this was not a goal of the original study. The heterogenous populations and varied aims and methodology of the included studies were well suited to an exploratory qualitative approach.

Regarding limitations, the findings of this review may not be generalisable given the relatively small number of studies and the importance of location and population to understanding concepts of climate change anxiety that emerged in the analysis. The exclusion of grey literature and other unpublished papers may have limited the results through publication bias [78]. The terms used to identify the spectrum of anxiety responses were not exhaustive; for example, the word "concern" was excluded from the literature search as it returned a prohibitive number of titles for review (over 25,000). Finally, the distinction between climate change anxiety and climate change worry was not always clearly delineated, both within the individual reviewed studies and consequently in the broader thematic analysis of the systematic review. This highlights the need for clarity and standardization of the concept of climate change anxiety.

\section{Conclusions}

Climate change anxiety is becoming recognised as one of the mental health effects of climate change. This review has identified a broad scope of worries about climate change, and a diversity of responses to this anxiety. Characteristics, such as occupation, socio-economic status, and proximity to climate change, appear to be important influences on climate change anxiety and related responses. The review furthers our understanding of the concept of climate change anxiety and highlights the need for future studies of this phenomenon to be conducted by clinical researchers. There is a pressing need to better understand the psychological and functional effects of climate change anxiety and to examine interventions to promote resilience and reduce clinically significant distress.

Author Contributions: Conceptualization, C.S. and A.P.F.W.; methodology, C.S. and A.P.F.W.; validation, A.P.F.W.; formal analysis, C.S. and A.P.F.W.; investigation, C.S.; writing-original draft preparation, C.S.; writing-review and editing, A.P.F.W.; visualization, C.S.; supervision, A.P.F.W.; project administration, C.S. and A.P.F.W. All authors have read and agreed to the published version of the manuscript.

Funding: This research did not receive any specific grant from funding agencies in the public, commercial, or not-for-profit sectors. This research received no external funding.

Institutional Review Board Statement: Not applicable.

Informed Consent Statement: Not applicable. 
Data Availability Statement: Not applicable.

Conflicts of Interest: The authors declare no conflict of interest.

\section{References}

1. Costello, A.; Abbas, M.; Allen, A.; Ball, S.; Bell, S.; Bellamy, R.; Friel, S.; Groce, N.; Johnson, A.; Kett, M. Managing the health effects of climate change: Lancet and University College London Institute for Global Health Commission. Lancet 2009, 373, 1693-1733. [CrossRef]

2. Berry, H.L.; Bowen, K.; Kjellstrom, T. Climate change and mental health: A causal pathways framework. Int. J. Public Health 2010, 55, 123-132. [CrossRef]

3. Fritze, J.G.; Blashki, G.A.; Burke, S.; Wiseman, J. Hope, despair and transformation: Climate change and the promotion of mental health and wellbeing. Int. J. Ment. Health Syst. 2008, 2, 13. [CrossRef]

4. Obradovich, N.; Migliorini, R.; Paulus, M.P.; Rahwan, I. Empirical evidence of mental health risks posed by climate change. Proc. Natl. Acad. Sci. USA 2018, 115, 10953-10958. [CrossRef]

5. Albrecht, G.; Sartore, G.-M.; Connor, L.; Higginbotham, N.; Freeman, S.; Kelly, B.; Stain, H.; Tonna, A.; Pollard, G. Solastalgia: The distress caused by environmental change. Australas. Psychiatry 2007, 15, S95-S98. [CrossRef]

6. Askland, H.H.; Bunn, M. Lived experiences of environmental change: Solastalgia, power and place. Emot. Space Soc. 2018, 27, 16-22. [CrossRef]

7. Galway, L.P.; Beery, T.; Jones-Casey, K.; Tasala, K. Mapping the solastalgia literature: A scoping review study. Int. J. Environ. Res. Public Health 2019, 16, 2662. [CrossRef]

8. Cunsolo, A.; Ellis, N.R. Ecological grief as a mental health response to climate change-related loss. Nat. Clim. Change 2018, 8 , 275-281. [CrossRef]

9. Cunsolo, A.; Harper, S.L.; Minor, K.; Hayes, K.; Williams, K.G.; Howard, C. Ecological grief and anxiety: The start of a healthy response to climate change? Lancet Planet. Health 2020, 4, e261-e263. [CrossRef]

10. Clayton, S.; Manning, C.; Krygsman, K.; Speiser, M. Mental Health and Our Changing Climate: Impacts, Implications, and Guidance, American Psychological Association and ecoAmerica: Washington, DC, USA, 2017.

11. Pihkala, P. Eco-Anxiety, Tragedy, and Hope: Psychological and Spiritual Dimensions of Climate Change. Zygon 2018, 53, 545-569. [CrossRef]

12. Clayton, S. Climate anxiety: Psychological responses to climate change. J. Anxiety Disord. 2020, 74, 102263. [CrossRef]

13. Clayton, S.; Karazsia, B.T. Development and validation of a measure of climate change anxiety. J. Environ. Psychol. 2020, 69, 101434. [CrossRef]

14. Stewart, A.E. Psychometric properties of the climate change worry scale. Int. J. Environ. Res. Public Health 2021, 18, 494. [CrossRef]

15. Taylor, M.; Murray, J. 'Overwhelming and Terrifying': The Rise of Climate Anxiety. Available online: https://www.theguardian. com/environment/2020/feb/10/overwhelming-and-terrifying-impact-of-climate-crisis-on-mental-health (accessed on 2 February 2021).

16. Ward, M. Climate Anxiety Is Real, and Young People Are Feeling It. Available online: https://www.smh.com.au/lifestyle/healthand-wellness / climate-anxiety-is-real-and-young-people-are-feeling-it-20190918-p52soj.html (accessed on 2 February 2021).

17. Crabb, A. Australia Talks National Survey Reveals What Australians Are Most Worried about. Available online: https:/ / www.abc.net.au/news/2019-10-08/annabel-crabb-australia-talks-what-australians-worry-about/11579644 (accessed on 2 February 2021).

18. ReachOut. New Survey by ReachOut and Student Edge Reveals Number of Students Anxious about Climate Change. Available online: https:/ / about.au.reachout.com/blog/new-survey-by-reachout-and-student-edge-reveals-number-of-students-anxiousabout-climate-change (accessed on 2 February 2021).

19. Searle, K.; Gow, K. Do concerns about climate change lead to distress? Int. J. Clim. Change Strateg. Manag. 2010, 2, 362-379. [CrossRef]

20. Corbin, J.; Strauss, A. Basics of Qualitative Research: Techniques and Procedures for Developing Grounded Theory; Sage Publications: Southend Oaks, CA, USA, 2014.

21. Attree, P.; Milton, B. Critically appraising qualitative research for systematic reviews: Defusing the methodological cluster bombs. Evid. Policy A J. Res. Debate Pract. 2006, 2, 109-126. [CrossRef]

22. Braun, V.; Clarke, V. Using thematic analysis in psychology. Qual. Res. Psychol. 2006, 3, 77-101. [CrossRef]

23. Anghelcev, G.; Chung, M.-Y.; Sar, S.; Duff, B.R. A ZMET-based analysis of perceptions of climate change among young South Koreans: Implications for social marketing communication. J. Soc. Mark. 2015, 5, 56-82. [CrossRef]

24. Du Bray, M.V.; Wutich, A.; Brewis, A. Hope and Worry: Gendered Emotional Geographies of Climate Change in Three Vulnerable US Communities. Weather. Clim. Soc. 2017, 9, 285-297. [CrossRef]

25. Fleming, A.; Dowd, A.-M.; Gaillard, E.; Park, S.; Howden, M. "Climate change is the least of my worries": Stress limitations on adaptive capacity. Rural Soc. 2015, 24, 24-41. [CrossRef]

26. Galway, L.P. Perceptions of climate change in Thunder Bay, Ontario: Towards a place-based understanding. Local Environ. 2019, 24, 68-88. [CrossRef] 
27. Gibson, K.; Barnett, J.; Haslam, N.; Kaplan, I. The mental health impacts of climate change: Findings from a Pacific Island atoll nation. J. Anxiety Disord. 2020, 73, 102237. [CrossRef]

28. Hoggett, P.; Randall, R. Engaging with climate change: Comparing the cultures of science and activism. Environ. Values 2018, 27, 223-243. [CrossRef]

29. Howard, M.; Ahmed, S.; Lachapelle, P.; Schure, M.B. Farmer and rancher perceptions of climate change and their relationships with mental health. J. Rural. Ment. Health 2020, 44, 87. [CrossRef]

30. Kemkes, R.J.; Akerman, S. Contending with the nature of climate change: Phenomenological interpretations from northern Wisconsin. Emot. Space Soc. 2019, 33, 100614. [CrossRef]

31. Norgaard, K.M. "People want to protect themselves a little bit": Emotions, denial, and social movement nonparticipation. Sociol. Inq. 2006, 76, 372-396. [CrossRef]

32. Nyantakyi-Frimpong, H.; Bezner-Kerr, R. The relative importance of climate change in the context of multiple stressors in semi-arid Ghana. Glob. Environ. Change 2015, 32, 40-56. [CrossRef]

33. Petheram, L.; Zander, K.K.; Campbell, B.M.; High, C.; Stacey, N. 'Strange changes': Indigenous perspectives of climate change and adaptation in NE Arnhem Land (Australia). Glob. Environ. Change 2010, 20, 681-692. [CrossRef]

34. Ryghaug, M.; Holtan Sørensen, K.; Næss, R. Making sense of global warming: Norwegians appropriating knowledge of anthropogenic climate change. Public Underst. Sci. 2011, 20, 778-795. [CrossRef]

35. Wright, C.; Nyberg, D. Working with passion: Emotionology, corporate environmentalism and climate change. Hum. Relat. 2012, 65, 1561-1587. [CrossRef]

36. Du Bray, M.V.; Wutich, A.; Larson, K.L.; White, D.D.; Brewis, A. Emotion, coping, and climate change in island nations: Implications for environmental justice. Environ. Justice 2017, 10, 102-107. [CrossRef]

37. Ojala, M. Regulating Worry, Promoting Hope: How Do Children, Adolescents, and Young Adults Cope with Climate Change? Int. J. Environ. Sci. Educ. 2012, 7, 537-561.

38. Bellamy, R.; Hulme, M. Beyond the tipping point: Understanding perceptions of abrupt climate change and their implications. Weather. Clim. Soc. 2011, 3, 48-60. [CrossRef]

39. Bouman, T.; Verschoor, M.; Albers, C.J.; Böhm, G.; Fisher, S.D.; Poortinga, W.; Whitmarsh, L.; Steg, L. When worry about climate change leads to climate action: How values, worry and personal responsibility relate to various climate actions. Glob. Environ. Change 2020, 62, 102061. [CrossRef]

40. Chu, H.; Yang, J.Z. Emotion and the psychological distance of climate change. Sci. Commun. 2019, 41, 761-789. [CrossRef]

41. Ekholm, S.; Olofsson, A. Parenthood and worrying about climate change: The limitations of previous approaches. Risk Anal. 2017, 37, 305-314. [CrossRef] [PubMed]

42. Fløttum, K.; Dahl, T.; Rivenes, V. Young Norwegians and their views on climate change and the future: Findings from a climate concerned and oil-rich nation. J. Youth Stud. 2016, 19, 1128-1143. [CrossRef]

43. Gareau, B.J.; Huang, X.; Gareau, T.P. Social and ecological conditions of cranberry production and climate change attitudes in New England. PLoS ONE 2018, 13, e0207237. [CrossRef]

44. Pfautsch, S.; Gray, T. Low factual understanding and high anxiety about climate warming impedes university students to become sustainability stewards: An Australian case study. Int. J. Sustain. High. Educ. 2017, 18, 1157-1175. [CrossRef]

45. Reser, J.; Bradley, G.; Glendon, A.; Ellul, M.; Callaghan, R. Public Risk Perceptions, Understandings, and Responses to Climate Change and Natural Disasters in Australia and Great Britain; National Climate Change Adaptation Research Facility: Gold Coast, Australia, 2012.

46. Verplanken, B.; Roy, D. “My worries are rational, climate change is not": Habitual ecological worrying is an adaptive response. PLOS ONE 2013, 8, e74708. [CrossRef]

47. Bilgin, A.; Balkaya, N. An assessment of the perspectives of university students on environmental issues in Turkey. Fresenius Environ. Bull. 2017, 26, 5271-5282.

48. Çimen, O.; Yılmaz, M. Environmental worries of teacher trainees. Procedia-Soc. Behav. Sci. 2011, 15, 2214-2218. [CrossRef]

49. Karatekin, K. Perception of environmental problem in elementary students' mind maps. Procedia-Soc. Behav. Sci. 2013, 93, 868-872. [CrossRef]

50. Ojala, M. Coping with climate change among adolescents: Implications for subjective well-being and environmental engagement. Sustainability 2013, 5, 2191-2209. [CrossRef]

51. Furberg, M.; Evengård, B.; Nilsson, M. Facing the limit of resilience: Perceptions of climate change among reindeer herding Sami in Sweden. Glob. Health Action 2011, 4, 8417. [CrossRef] [PubMed]

52. Barnett, J.; Adger, W.N. Climate dangers and atoll countries. Clim. Change 2003, 61, 321-337. [CrossRef]

53. Alonso, A.D.; O'Neill, M.A. Climate change from the perspective of Spanish wine growers: A three-region study. Br. Food J. 2011, 113, 205-221. [CrossRef]

54. Lewis, G.B.; Palm, R.; Feng, B. Cross-national variation in determinants of climate change concern. Environ. Politics 2019, 28, 793-821. [CrossRef]

55. Muttarak, R.; Chankrajang, T. Who is concerned about and takes action on climate change? Gender and education divides among Thais. Vienna Yearb. Popul. Res. 2015, 13, 193-220. [CrossRef]

56. Sundblad, E.-L.; Biel, A.; Gärling, T. Cognitive and affective risk judgements related to climate change. J. Environ. Psychol. 2007, 27, 97-106. [CrossRef] 
57. Wolf, J.; Moser, S.C. Individual understandings, perceptions, and engagement with climate change: Insights from in-depth studies across the world. Wiley Interdiscip. Rev. Clim. Change 2011, 2, 547-569. [CrossRef]

58. Bradley, G.L.; Reser, J.P.; Glendon, A.I.; Ellul, M.C. Distress and coping in response to climate change. In Stress and Anxiety: Applications to Social and Environmental Threats, Psychological Well-Being, Occupational Challenges, and Developmental Psychology Climate Change; Logos Verlag Berlin: Berlin, Germany, 2014; pp. 33-42.

59. Ojala, M. How do children cope with global climate change? Coping strategies, engagement, and well-being. J. Environ. Psychol. 2012, 32, 225-233. [CrossRef]

60. Hayes, K.; Blashki, G.; Wiseman, J.; Burke, S.; Reifels, L. Climate change and mental health: Risks, impacts and priority actions. Int. J. Ment. Health Syst. 2018, 12, 28. [CrossRef]

61. Li, C.J.; Monroe, M.C. Exploring the essential psychological factors in fostering hope concerning climate change. Environ. Educ. Res. 2019, 25, 936-954. [CrossRef]

62. Mineka, S.; Watson, D.; Clark, L.A. Comorbidity of anxiety and unipolar mood disorders. Annu. Rev. Psychol. 1998, 49, 377-412. [CrossRef] [PubMed]

63. McNamara, K.E.; Westoby, R. Solastalgia and the gendered nature of climate change: An example from Erub Island, Torres Strait. EcoHealth 2011, 8, 233-236. [CrossRef]

64. Willox, A.C.; Harper, S.L.; Edge, V.L.; Landman, K.; Houle, K.; Ford, J.D. The land enriches the soul: On climatic and environmental change, affect, and emotional health and well-being in Rigolet, Nunatsiavut, Canada. Emot. Space Soc. 2013, 6, 14-24. [CrossRef]

65. Haseley, D. Climate change: Clinical considerations. Int. J. Appl. Psychoanal. Stud. 2019, 16, 109-115. [CrossRef]

66. Jones, M.K.; Wootton, B.M.; Vaccaro, L.D.; Menzies, R.G. The impact of climate change on obsessive compulsive checking concerns. Aust. N. Z. J. Psychiatry 2012, 46, 265-270. [CrossRef]

67. Baudon, P.; Jachens, L. A scoping review of interventions for the treatment of eco-anxiety. Int. J. Environ. Res. Public Health 2021, 18, 9636. [CrossRef]

68. Berry, H.L.; Peel, D. Worrying about climate change: Is it responsible to promote public debate? BJPsych Int. 2015, 12, 31-32 [CrossRef]

69. Helm, S.V.; Pollitt, A.; Barnett, M.A.; Curran, M.A.; Craig, Z.R. Differentiating environmental concern in the context of psychological adaption to climate change. Glob. Environ. Change 2018, 48, 158-167. [CrossRef]

70. Temte, J.L.; Holzhauer, J.R.; Kushner, K.P. Correlation between climate change and dysphoria in primary care. WMJ 2019, 118, 71-74.

71. Higginbotham, N.; Connor, L.; Albrecht, G.; Freeman, S.; Agho, K. Validation of an environmental distress scale. EcoHealth 2006, 3, 245-254. [CrossRef]

72. Du Bray, M.; Wutich, A.; Larson, K.L.; White, D.D.; Brewis, A. Anger and sadness: Gendered emotional responses to climate threats in four island nations. Cross-Cult. Res. 2019, 53, 58-86. [CrossRef]

73. Becken, S.; Lama, A.K.; Espiner, S. The cultural context of climate change impacts: Perceptions among community members in the Annapurna Conservation Area, Nepal. Environ. Dev. 2013, 8, 22-37. [CrossRef]

74. Iniguez-Gallardo, V.; Bride, I.; Tzanopoulos, J. Between concepts and experiences: Understandings of climate change in southern Ecuador. Public Underst. Sci. 2020, 29, 745-756. [CrossRef] [PubMed]

75. Kittipongvises, S.; Mino, T. The influence of psychological factors on global climate change perceptions held by the rural citizens of Thailand. Ecopsychology 2013, 5, 126-135. [CrossRef]

76. Moghariya, D.P.; Smardon, R.C. Rural perspectives of climate change: A study from Saurastra and Kutch of Western India. Public Underst. Sci. 2014, 23, 660-677. [CrossRef]

77. Maran, D.A.; Begotti, T. Media Exposure to Climate Change, Anxiety, and Efficacy Beliefs in a Sample of Italian University Students. Int. J. Environ. Res. Public Health 2021, 18, 9358. [CrossRef]

78. Easterbrook, P.J.; Gopalan, R.; Berlin, J.; Matthews, D.R. Publication bias in clinical research. Lancet 1991, 337, 867-872. [CrossRef] 